\title{
Quantum processes on phase space
}

\author{
Charis Anastopoulos, \\ Spinoza Instituut, Leuvenlaan 4, \\ 3584HE Utrecht, The Netherlands
}

October 26, 2018

\begin{abstract}
Quantum theory predicts probabilities for various events as well as relative phases (interference or geometric) between different alternatives of the system. The most general description of the latter is in terms of the Pancharatnam phase. A unified description of both probabilities and phases comes through generalisation of the notion of a density matrix for histories; this object is the decoherence functional introduced by the consistent histories approach. If we take phases as well as probabilities as primitive elements of our theory, we abandon Kolmogorov probability and can describe quantum theory in terms of fundamental commutative observables, without being obstructed by Bell's and related theorems.

We develop the description of relative phases and probabilities for paths on the classical phase space. This description provides a theory of quantum processes, having many formal analogies with the theory of stochastic processes. We identify a number of basic postulates and study its corresponding properties. We strongly emphasise the notion of conditioning (by generalising classical conditional probability) and are able to write "quantum differential equations" as analogous to stochastic differential equations. They can be interpreted as referring to individual systems.

We, then, show the sense by which quantum theory is equivalent to a quantum process on phase space (using coherent states). Conversely starting from quantum processes on phase space we recover standard quantum theory on Hilbert space from the requirement that the process satisfies (an analogue of ) the Markov property together with time reversibility. The statistical predictions of our theory are identical to the ones of standard quantum theory, but the "logic" of events is Boolean; events are not represented by projectors any more. We discuss some implication of this fact for the interpretation of quantum theory, emphasising that it makes plausible the existence of realist theories for individual quantum systems.
\end{abstract}

*anastop@phys.uu.nl 


\section{Introduction}

\subsection{Quantum phases and probabilities}

Quantum mechanics is a theory of probabilities, but it is not a probability theory, in the standard use of this term. The reason for this lies in the fact that the basic objects in quantum theory are vectors in a complex Hilbert space (wave functions) and probabilities are obtained by squaring them. Hence, the basic quantum object is the probability amplitude, not the probability itself.

When we deal with properties of systems at a single moment of time, this distinction loses some of its immediacy. Indeed, one can describe quantum theory in terms of states (density matrices), observables (self-adjoint operators) and propositions (projection operators): these concepts are identical to the ones employed by classical probability theory. The only difference lies in the noncommutativity of the space of observables.

However, this exact correspondence evaporates when we consider the study of properties of the system at more than one moments of time. In this case the underlying complex nature of the basic quantities manifests itself in the presence of phases, either ones associated to interference or geometric ones.

The two-slit experiment is well known as a prototype of quantum mechanical interference. It is a special case of a general mathematical property of quantum theory: probabilities corresponding to histories are non-additive, i.e. if we have two histories (propositions) $\alpha$ and $\beta$ then

$$
p(\alpha \vee \beta) \neq p(\alpha)+p(\beta)
$$

Here $p$ refers to the probability distribution associated to a state and $\vee$ refers to the conjunction of histories viewed as propositions.

This fact is the starting point of the consistent histories approach $[1,2,3$, $4,5]$, which is a realist interpretation of quantum theory. It remarks that the probabilistic information about histories can be incorporated into a function of pairs of histories, the decoherence functional. Formally, this is a generalisation of the notion of the density matrix in the history context. Whenever we have a set of histories in which the off-diagonal elements of the decoherence functional are vanishing, its diagonal elements can be unambiguously interpreted as a probability distribution for this set of histories. However the consistent histories approach does not offer a natural physical interpretation for the off-diagonal elements of the decoherence functional.

Besides the non-additivity of probabilities the complex nature of quantum theory manifests itself in the importance of geometric phases, i.e. phases that appear during the quantum evolution of the system and depend only on the path transversed and not on the duration of the evolution or the Hamiltonian that drives the system. The paradigmatic example is the Berry phase $[6,7$, 8], which was originally derived for cyclic, adiabatic evolution. However, it was soon realised that the geometric phase exists for non-cyclic, non-adiabatic 
evolutions [9] and, what is important for our present context, for sequences of measurements [10]. An important property of the geometric phase is that it can be measured only relatively, i.e. by comparing the evolution of two distinct ensembles of systems through their interference pattern.

The geometric phase and the histories approach can be viewed in a unified framework by noticing that the geometric phase is the building block of the decoherence functional. In [11] it was shown that for the limit of continuous-time histories the off-diagonal elements of the decoherence functional amount to the difference in geometric phase between the paths, thus providing an interpretation lacking in the consistent histories approach. This interpretation persists for the case of coarse-grained histories at discrete moments of time, by virtue of the generalisations of the geometric phase [12]. We show in this paper (section 2.2 ) that the most natural interpretation of the off-diagonal elements of the decoherence functional is in terms of the geometric phase that arose out of the work of Pancharatnam [13, 10].

The picture that emerges from these results is that a formalism for quantum theory that is based on histories, needs to include not only the notion of probabilities, but also that of the relative phases. If we take the phases as elementary ingredients of the theory we abandon the idea that quantum theory is to be based on additive probabilities. But then the classic non-go theorems of Bell-Wigner [14] or Kochen-Specker [15] are not forbidding for the description of the quantum system in terms of some version of "hidden variables", that are not deterministic. In [12] we showed that one can recover all statistical predictions of quantum theory (essentially the correlation functions) from a theory that is based on commutative observables, essentially functions on the classical phase space. The only difference is in the notion of events: in quantum theory events are said to correspond to projection operators (and hence form a non-distributive lattice), while in our construction they correspond to subsets of phase space and as such they form a classical Boolean lattice.

\subsection{This paper}

An epigrammatic way to state our previous discussion is that if we treat quantum phases as basic ingredients of quantum theory allows us to get the predictions of standard quantum theory, while dispensing with quantum logic.

This statement is the starting point of this paper. We want to develop a statistical theory for histories on a manifold (usually a symplectic manifold), which will be based on a decoherence functional rather than a probability measure. We take as prototype the theory of stochastic processes, and for this reason we call this class of theoriesquantum processes. We need to warn the reader that this name is not used in the same sense as has been used in the literature, where it was used to denote stochastic processes on the quantum mechanical Hilbert space (see [16] for a recent review).

Two are the main ingredients of the theory of quantum processes. One 
first needs to specify, what the events are. We take an elementary event to correspond to a point of a sample space $\Omega$ and a (coarse-grained) event is a measurable subset of $\Omega$. In the context of histories $\Omega$ is taken as a space of suitable maps from a subset of the real line $\mathbf{R}$ to a manifold $\Gamma$. We shall mostly identify $\Gamma$ with the classical phase space.

The other ingredient is the decoherence functional: it is a complex-valued functional that takes a pair of histories to a complex number in the unit disc. Its defining properties are essentially these of a density matrix; in fact they have a direct operational significance, in terms of the standard procedure of measuring the relative phase between different histories of the system. The only condition without a direct operational significance is the additivity, which is a manifestation of the superposition principle of standard quantum theory. When we have histories, we can define the decoherence functional in terms of its restriction on discrete moments of time. In a fashion similar to classical probability theory, a decoherence functional on continuous-time histories can be defined by a hierarchy of multi-time hierarchy distributions that satisfy a condition of compatibility.

Again in analogy to classical probability theory, we introduce the notion of conditioning, i.e. the changing of the physical quantities in such a way as to take into account the fact that we focus on a particular class of observables. Conditioning takes place with respect to a subalgebra of observables, which correspond to the particular class of events to which we focus our description. The standard result of the "reduction of the wave packet" arises as a special case.

Now, whenever we have histories there is a sharp distinction between the notion of time as manifested in kinematics as compared with the way it is manifested in dynamics. This was shown by Savvidou [17], in the context of the continuous-time formulation of consistent histories, but makes sense in any theory that has histories as basic objects (whether this is quantum theory or classical mechanics or the theory of stochastic processes). In effect, the histories description allows a distinction between the kinematical and dynamical aspects of time, which is implemented by different symmetry groups $[18,19]$. In quantum theory, it is argued that this distinction manifests itself in the distinction between "reduction of the wave packet" and Heisenberg dynamics [17] or between the geometric and dynamical phases [11].

For this reason it is convenient to distinguish between the kinematical and dynamical aspects of quantum processes; we distinguish the kinematic process, which is obtained for vanishing Hamiltonian: this is unlike classical probability theory, where the kinematic processes are trivial; in quantum theory the kinematic process contains all characteristic features of quantum theory. We construct such processes for standard quantum theory, by employing coherent states. The introduction of dynamics is then rather straightforward; one can write quantum differential equations in analogy with stochastic differential equations, in which "fluctuating forces" subject to the kinematical process com- 
plement deterministic evolution. The kinematic process is then a building block for quantum processes: in effect it is the analogue of the Wiener process in the theory of stochastic processes, which is usually employed in order to model a fluctuating environment.

We then proceed to argue inversely: given a quantum process on phase space, how do we recover standard quantum theory? The answer is very simple: if we assume that the process satisfies an analogue of the Markov property and time-reversibility, then this process can be described by quantum theory on the standard Hilbert space. Dropping time-reversibility one gets the theory of open quantum systems. It is, however, important to make an assumption of continuity for the propagator, which amounts to demanding the existence of a non-trivial kinematic process associated to the system under study.

Eventually, we arrive at the point we aimed to: a theory that has the same statistical predictions of quantum theory, but whose notion of events is different; an event (or a sharp measurement) is not associated to a projection operator, but to a phase space cell. Hence the "logic" of these events is Boolean. In comparing with standard quantum theory, this means that we drop the principle that measurable quantities correspond to eigenvalues of the operator observables. We argue that this principle has actually no compelling experimental evidence and is rather made for historical reasons and for purposes of convenience. The discreteness manifested in many quantum phenomena can eventually be attributed to dynamics or a combination of dynamics and kinematics. After all, this kind of information is contained in the correlation functions, which are obtainable by a quantum process, without any assumption about a special status of operators' eigenvalues.

Our analysis demonstrates, that it is possible to set theoretical frameworks that can provide the same statistical predictions to the ones of quantum theory, while having as observables only real-valued functions. Quantum theory is then possible without "quantum logic". Even though our construction is operational in character and cannot claim to be a theory for the individual quantum system (like Kopenhagen quantum theory), it is not incompatible with such a theory. And by virtue of the preservation of classical logic no issues of contextuality -like one exhibited in the Kochen-Specker "paradox"- are likely to arise.

Throughout the paper there are three points, the consequences of which we invariably point out:

i. The formal analogy of quantum processes to classical stochastic processes, that allows us to employ successful techniques from the latter theory in the former. For this paper we have tried to keep the perspective on the rigorous presentation as in [20], but we have found particularly useful the semi-formal treatment of [21] and the perspective afforded by [16, 22].

ii. The fact that the fundamental behaviour of quantum theory is already present at the kinematical level. In particular, the presence of complex num- 
bers as manifested in the phases is not due to the Schrödinger equation as often assumed, but goes deeper in the fundamental set up of the theory.

iii. Our eventual aim is to find a description for the individual system, that would reproduce the description in terms of quantum processes in the statistics. We have reasons to believe that such a description might be possible to be based in the geometry of the classical phase space, or rather on additional geometric structures introduced in the classical phase space. For this reason, we give some emphasis in possible geometric origins of our basic objects.

\section{Background}

\subsection{The standard histories formalism}

A history corresponds to a sequence of projection operators $\hat{\alpha}_{t_{1}}, \ldots, \hat{\alpha}_{t_{n}}$, and it corresponds to a time-ordered sequence of propositions about the physical system. The indices $t_{1}, \ldots, t_{n}$ refer to the time a proposition is asserted and have no dynamical meaning. Dynamics are related to the Hamiltonian $\hat{H}$, which defines the one-parameter group of unitary operators $\hat{U}(s)=e^{-i \hat{H} s}$.

A natural way to represent the space of all histories is by defining a history Hilbert space $\mathcal{V}:=\otimes_{t_{i}} \mathcal{H}_{t_{i}}$, where $\mathcal{H}_{t_{i}}$ is a copy of the standard Hilbert space, indexed by the moment of time to which it corresponds. A history is then represented by a projection operator on $\mathcal{V}$. This construction has the merit of preserving the quantum logic structure [5] and highlighting the non-trivial temporal structure of histories $[17,23]$. Furthermore, one can also construct a Hilbert space $\mathcal{V}$ for continuous-time histories $[24,25,26]$ by a suitable definition of the notion of the tensor product.

Furthermore, to each history $\alpha$ we may associate the class operator $\hat{C}_{\alpha}$ defined by

$$
\hat{C}_{\alpha}=\hat{U}^{\dagger}\left(t_{n}\right) \hat{\alpha}_{t_{n}} \hat{U}\left(t_{n}\right) \ldots \hat{U}^{\dagger}\left(t_{1}\right) \hat{\alpha}_{t_{1}} \hat{U}\left(t_{1}\right) .
$$

The decoherence functional is defined as a complex-valued function of pairs of histories: i.e. a map $d: \mathcal{V} \times \mathcal{V} \rightarrow$ C. For two histories $\alpha$ and $\alpha^{\prime}$ it is given by

$$
d\left(\alpha, \alpha^{\prime}\right)=\operatorname{Tr}\left(\hat{C}_{\alpha} \hat{\rho}_{0} \hat{C}_{\alpha^{\prime}}^{\dagger}\right)
$$

The consistent histories interpretation of this object is that when $d\left(\alpha, \alpha^{\prime}\right)=0$ for $\alpha \neq \alpha^{\prime}$ in an exhaustive and exclusive set of histories ${ }^{1}$, then one may assign a probability distribution to this set as $p(\alpha)=d(\alpha, \alpha)$. The value of $d(\alpha, \beta)$ is, therefore, a measure of the degree of interference between the histories $\alpha$ and $\beta$.

\footnotetext{
${ }^{1}$ By exhaustive we mean that at each moment of time $t_{i}, \sum_{\hat{\alpha}_{t_{i}}} \hat{\alpha}_{t_{i}}=1$ and by exclusive that $\hat{\alpha}_{t_{i}} \hat{\beta}_{t_{i}}=\delta_{\alpha \beta}$. Note that by $\alpha$ we denote the proposition with the corresponding projector written as $\hat{\alpha}$ with a hat.
} 
But one can view the decoherence functional solely in an operational perspective. The state $\hat{\rho}_{0}$ corresponds to a preparation of an ensemble of systems. This can be visualised as a beam of particles, which passes through various filters in a succession. The filters correspond to the projection operators that constitute the history; the diagonal elements of the decoherence functional gives the intensity of the beam that has passed through these filters. There is no a priori reason for these intensities to be described by an additive probability measure. The decoherence condition can be taken as specifying the domain of validity of an approximation, by which the full quantum mechanical description is substituted by an effective one through probability theory [27]. However, even the operational description does not explain the physical meaning of the off-diagonal elements of the decoherence functional.

\subsection{Interpretation of the off-diagonal elements}

The interpretation of the off-diagonal elements of the decoherence functional is more conveniently carried out in terms of a version of the geometric phase, know as the Pancharatnam phase. Its origin lies in the following considerations.

A quantum state is specified by a normalised Hilbert space vector, up to a phase. In other words, a (pure) quantum state corresponds to an element of the projective Hilbert space $P H$. In fact, the unit sphere in the Hilbert space forms a $U(1)$ principal fiber bundle over $P H$, known as the Hopf bundle. The absolute phase of a vector then makes no physical sense, but it is desirable to be able to compare the relative phase between two different vectors.

Consider two normalised vectors $|\psi\rangle$ and $|\phi\rangle$ and perform the operation of changing the phase of $|\psi\rangle$ by a factor $e^{i \chi}$. Interfering $e^{i \chi}|\psi\rangle$ and $|\phi\rangle$, we get a beam with intensity

$$
\| e^{i \chi}|\psi\rangle+|\phi\rangle \|^{2}=2+2|\langle\psi \mid \phi\rangle| \cos (\chi-\arg \langle\psi \mid \phi\rangle)
$$

This intensity achieves a maximum for $\chi=\arg \langle\psi \mid \phi\rangle$. This value for $\chi$ is the Pancharatnam phase between the two vectors $|\psi\rangle$ and $|\phi\rangle$. It has been experimentally determined in certain occasions (see [28] for a measurement using neutron interferometry): one needs to implement the transformations $|\psi\rangle \rightarrow e^{i \chi}|\psi\rangle$ (e.g through the action of the Hamiltonian of which it $|\psi\rangle$ is an eigenstate) and then find the maximum of the intensity of the combined beam. We should note that the Pancharatnam phase has a close mathematical relationship with the natural connection on the Hilbert's space Hopf bundle [10].

Consider, now, the simplest example of a history. We have a beam of particles characterised by the Hilbert space vector $|\psi\rangle$ and at times $t_{1}$ and $t_{2}$ measurements corresponding to (generally non-commuting operators) $\hat{\alpha}_{1}$ and $\hat{\alpha}_{2}$ respectively. Let us absorb the Hamiltonian evolution in a redefinition of the projection operators. The beam passing through the projectors will be $\hat{\alpha}_{2} \hat{\alpha}_{1}|\psi\rangle$. It is clear that the value of the decoherence functional between the history $\alpha$ 
(that the system passed through $\alpha_{1}$ and $\alpha_{2}$ successively) and the trivial history 1 (i.e. no measurement on $|\psi\rangle$ ) will be the Pancharatnam phase between $|\psi\rangle$ and $\hat{\alpha}_{2} \hat{\alpha}_{1}|\psi\rangle$.

Let us be more precise. The number $\left\langle\psi\left|\hat{\alpha}_{1} \hat{\alpha}_{2} \hat{\alpha}_{2} \hat{\alpha}_{1}\right| \psi\right\rangle$ can be determined by a measurement of the intensity of $\hat{\alpha}_{2} \hat{\alpha}_{1}|\psi\rangle$. It is essentially equal to the diagonal element of the decoherence functional $d(\alpha, \alpha)=r$. We want to measure $d(\alpha, 1)=\left\langle\psi\left|\hat{\alpha}_{s} \hat{\alpha}_{1}\right| \psi\right\rangle:=\rho e^{i \beta}$, where $0 \leq \rho \leq 1$.

Similarly to the previous discussion we interfere the beam $\hat{\alpha}_{2} \hat{\alpha}_{1}|\psi\rangle$, with $e^{i \chi}|\psi\rangle$. This yields the intensity

$$
I(\chi)=\| e^{i \chi}|\psi\rangle+\hat{\alpha}_{2} \hat{\alpha}_{1}|\psi\rangle \|^{2}=1+r^{2}+2 \rho \cos (\chi-\beta)
$$

This intensity has a maximum for $\chi=\beta$ : this determines $\arg d(\alpha, 1)$. At the maximum of the intensity we have $I_{\max }=1+r^{2}+2 \rho$. Since we know $r$ from a previous experiment and the value of $I_{\max }$ can be measured, we can determine $\rho$. Thus by measuring the intensity for different values of $\chi$, we have determined the off-diagonal element of the decoherence functional. Note, that the determination of this phase necessitates the study of the interference of two beams, as is always the case in the measurement of quantum phases.

This procedure can be easily repeated -in principle- for the interference of arbitrary pairs of histories. The off-diagonal elements of the decoherence functional will then always be determined through the measurement of the Pancharatnam phase between two vectors.

\subsection{Correlation functions}

Let us now consider an ensemble of quantum systems prepared in a state described by a density matrix $\hat{\rho}$ and try to operationally construct the correlation function of two observables $\hat{A}=\sum a_{i} \hat{\alpha}_{i}$ and $\hat{B}=\sum_{j} b_{j} \hat{\beta}_{j}$ at times $t_{1}$ and $t_{2}>t_{1}$ respectively. Here $\hat{\alpha}_{i}$ are an exhaustive and exclusive set of projectors, and so is $\hat{\beta}_{j}$.

Let the Hamiltonian of the system be $\hat{H}$ and $\hat{\rho}_{0}$ the state of the system at time $t=0$. The probability that both $\hat{\alpha}_{i}$ and then $\hat{\beta}_{j}$ are true will be

$$
\begin{array}{r}
p\left(i, t_{1} ; j, t_{2}\right)=\operatorname{Tr}\left(\hat{\beta}_{j} e^{-i \hat{H}\left(t_{2}-t_{1}\right)} \hat{\alpha}_{i} e^{-i \hat{H} t_{1}} \hat{\rho}_{0} e^{i \hat{H} t_{1}} \hat{\alpha}_{i} e^{i \hat{H}\left(t_{2}-t_{1}\right)}\right)= \\
\operatorname{Tr}\left(\hat{\beta}_{j}\left(t_{2}\right) \hat{\alpha}_{i}\left(t_{1}\right) \hat{\rho}_{0} \hat{\alpha}_{i}\left(t_{1}\right)\right),
\end{array}
$$

If we now vary over all possible values of $i$ and $j$, we can construct the statistical correlation function between $\hat{A}$ and $\hat{B}$

$$
\left\langle\hat{A}_{t_{1}} \hat{B}_{t_{2}}\right\rangle_{S}=\sum_{i j} a_{i} b_{j} p\left(i . t_{1} ; j, t_{2}\right)
$$

But this correlation function is not what one usually calls correlation function in quantum theory. This name is usually employed for the expectation of a 
product of operators

$$
\left\langle\hat{A}_{t_{1}} \hat{B}_{t_{2}}\right\rangle=\operatorname{Tr}\left(\hat{A}\left(t_{1}\right) \hat{B}\left(t_{2}\right) \hat{\rho}\right)=\sum_{i j} a_{i} b_{j} \operatorname{Tr}\left(\hat{\alpha}_{i}\left(t_{1}\right) \hat{\beta}_{j}\left(t_{2}\right) \hat{\rho}\right) .
$$

This is a complex-valued object, in contrast to (1.10) that was constructed using frequencies of events and can only be real-valued. What does then the quantum mechanical correlation correspond to? Our previous discussion makes it now clear. The complex-valued quantum mechanical correlation function is related to the off-diagonal elements of the decoherence functional and essentially contains information about relative phases.

The precise relation is as follows. Let $\hat{A}^{a}$ denote a family of commuting operators. Then the time-ordered two-point correlation function is defined as

$$
\begin{array}{r}
G^{2,0}\left(a_{1}, t_{1} ; a_{2}, t_{2}\right)=\theta\left(t_{2}-t_{1}\right) \operatorname{Tr}\left[\hat{A}^{a_{1}}\left(t_{1}\right) \hat{A}^{a_{2}}\left(t_{2}\right) \hat{\rho}_{0}\right]+ \\
\theta\left(t_{1}-t_{2}\right) \operatorname{Tr}\left[\hat{A}^{a_{2}}\left(t_{2}\right) \hat{A}^{a_{1}}\left(t_{1}\right) \hat{\rho}_{0}\right]
\end{array}
$$

One can similarly define time-ordered $n$-point functions, or anti-time-ordered

$$
\begin{array}{r}
G^{0,2}\left(a_{1}, t_{1} ; a_{2}, t_{2}\right)=\theta\left(t_{1}-t_{2}\right) \operatorname{Tr}\left[\hat{A}^{a_{1}}\left(t_{1}\right) \hat{A}^{a_{2}}\left(t_{2}\right) \hat{\rho}_{0}\right]+ \\
\theta\left(t_{2}-t_{1}\right) \operatorname{Tr}\left[\hat{A}^{a}\left(t_{2}\right) \hat{A}^{a_{1}}\left(t_{1}\right) \hat{\rho}_{0}\right]
\end{array}
$$

In general, one can define mixed correlation functions $G^{r, s}$, with $r$ time-ordered and $s$ anti-time-ordered entries, as for instance

$$
\begin{array}{r}
G^{2,1}\left(a_{1}, t_{1} ; a_{2}, t_{2} \mid b_{1}, t_{1}^{\prime}\right)=\theta\left(t_{2}-t_{1}\right) \operatorname{Tr}\left[\hat{A}^{a_{1}}\left(t_{1}\right) \hat{A}^{a_{2}}\left(t_{2}\right) \hat{\rho}_{0}\left[\hat{A}^{b_{1}}\left(t_{1}^{\prime}\right)\right]+\right. \\
\theta\left(t_{1}-t_{2}\right) \operatorname{Tr}\left[\hat{A}^{a_{2}}\left(t_{2}\right) \hat{A}^{a_{1}}\left(t_{1}\right) \hat{\rho}_{0} \hat{A}^{b_{1}}\left(t_{1}^{\prime}\right)\right]
\end{array}
$$

These correlation functions are generated by the Closed-Time-Path (CTP) generating functional associated to the family $\hat{A}^{a}$

$$
\begin{array}{r}
Z_{A}\left[J_{+}, J_{-}\right]= \\
\sum_{n, m=0}^{\infty} \frac{i^{n}(-i)^{m}}{n ! m !} \int d t_{1} \ldots d t_{n} d t_{1}^{\prime} \ldots d t_{m}^{\prime} \\
G^{n, m}\left(a_{1}, t_{1} ; \ldots a_{n}, t_{n} \mid b_{1}, t_{1}^{\prime} ; \ldots ; b_{m}, t_{m}^{\prime}\right) \\
J_{+}^{a_{1}}\left(t_{1}\right) \ldots J^{a_{n}}\left(t_{n}\right) J_{-}^{b_{1}}\left(t_{1}^{\prime}\right) \ldots J^{b_{m}}\left(t_{m}^{\prime}\right)
\end{array}
$$

The name closed-time arose, because in the original conception (by Schwinger [29] and Keldysh [30] the time path one follows is from some initial time $t=0$ to $t \rightarrow \infty$ moving in a time-ordered fashion and then back from infinity to 0 in an anti-time-ordered fashion. The total time-path is in effect closed.

Clearly there must be a relation between the decoherence functional and the CTP one. One can see in the correlation functions, if we assume a single operator $\hat{A}=\sum_{i} a_{i} \hat{\alpha}_{i}$ and consider a pair of histories $\alpha\left(i_{1}, t_{1} ; \ldots ; i_{n}, t_{n}\right)=$ 
$\left\{\hat{\alpha}_{i_{1}}, t_{1} ; \ldots ; \hat{\alpha}_{i_{n}}, t_{n}\right\}$ and $\alpha^{\prime}\left(i_{1}, t_{1}^{\prime} ; \ldots ; i_{n}, t_{m}^{\prime}\right)=\left\{\hat{\alpha}_{j_{1}}, t_{1}^{\prime} ; \ldots ; \hat{\alpha}_{j_{m}}, t_{m}\right\}$. Then one can verify $[26]$ that

$$
\begin{array}{r}
G_{A}^{n, m}\left(t_{1}, \ldots, t_{n} ; t_{1}^{\prime}, \ldots, t_{m}^{\prime}\right)=\sum_{i_{1} \ldots i_{n}} \sum_{j_{1} \ldots j_{m}} a_{i_{1}} \ldots a_{i_{n}} b_{j_{1}} \ldots b_{j_{m}} \\
\times d\left[\alpha\left(i_{1}, t_{1} ; \ldots ; i_{n}, t_{n}\right), \alpha^{\prime}\left(j_{1}, t_{1} ; \ldots ; j_{m}, t_{m}\right)\right]
\end{array}
$$

One needs to consider a decoherence functional for continuous-time histories $[24,25,17]$ and this requires a significant upgrading of the formalism of quantum mechanical histories. The key idea is to represent histories by projectors on a tensor product of Hilbert spaces $\otimes_{t \in T} H_{t}[5]$ in analogy to the construction of the history sample space classically. A suitable Hilbert space (not a genuine tensor product) can be constructed [24] for the case that $T$ is a continuous set and the decoherence functional can be defined as a bilinear, hermitian functional on this space. It can then be shown that as a functional it is essentially a double "Fourier transform" of the CTP generating functional [26]. This is a construction, we shall repeat in the context of the present paper.

\subsection{Commutative observables}

The study of quantum mechanical histories has shown that phases are equally important structural elements of quantum theory to probabilities and they are closely related to the non-additivity of the probability assignment of quantum theory. Moreover, there exists a well defined operational procedure by which they can be measured. Accepting phases as primitive elements of quantum theory allows us to phrase it in a way that is independent of Kolmogorov' probability or their generalisations.

Now the assumption of Kolmogorov probability is a crucial ingredient of (generalisations of) proofs of Bell's theorem against the possibility of local hidden variable theories. An assumption that follows from additive probability (namely that if an event has probability one its complement has probability zero) is employed in the Kochen-Specker's theorem against uncontextual assignment of properties in quantum systems. If the assumption of Kolmogorov probability is abandoned the way is open to write versions of quantum theory that contain local hidden-variable theories.

Such theories are, of course, non-deterministic and not probability theories in the classical sense. The behaviour of individual systems is "random" and ensembles exhibit behaviour that manifests in non-additive probabilities and relative phases. This information can be included in a version of the decoherence functional.

In effect, such are history theories (satisfying a modified form of the GellMann-Hartle-Isham axioms), which are described by commutative observables. The basic observables are then functions on some classical set, usually a manifold. In [12] we argued that the best choice for this base space would be the 
classical phase space. The reason is that the quantum mechanical Hilbert space typically contains the irreducible representation of a group, which also acts transitively on the classical phase space (see [32] for the general perspective on this quantum-symplectic correspondence). This provides a way (in fact more than one) to map quantum mechanical operators to functions on the phase space. A theory developed on phase space would then be able to include all information included in the Hilbert space operators. This we showed to be true using the Wigner transform.

The main difference of such theories from standard quantum mechanics lies in the notion of an event. In a classical space a (sharp) event corresponds to a subset of the phase space, while in quantum theory events correspond to projection operators. There exists no exact correspondence between these two objects; however the two theories give identical quantum mechanical correlation functions of the type (2. 7) and their difference is at the order of $\hbar$ in the statistical correlation functions (2. 6). We have not been able to find any experimental situation that would be able to unambiguously distinguish them.

In the following sections we shall give a more detailed construction of this class of theories.

\section{The basic structures}

We shall here provide the basic features of the class of theories we described earlier; they are based on the introduction of a decoherence functional for paths on the classical phase space. We shall draw analogies on the formal similarity with stochastic processes; for this reason we shall refer to our class of theories as theories of quantum processes.

\subsection{Events and observables}

At the level of observables, the structure of our theory is identical with that of classical probability theory. That is, we assume the existence of a space $\Omega$ of elementary alternatives. A point of $\Omega$ corresponds to the most precise information one can extract from a measurement of the quantum system. Note, that at this level we do not distinguish whether $\Omega$ refers to properties of a systems at one moment of time or to histories. Our definitions are general and only in the next section shall we specify the history content.

This space $\Omega$ has to be equipped with some additional structure. In general, a measurement will yield some information that the system was found in a given subset of $\Omega$. But not all subsets of $\Omega$ are suitable to incorporate measurement outcomes. For instance, when we consider position it is physically meaningless to consider the subset of rational values of position (with respect to some unit). One, therefore needs to choose a family of subsets $\mathcal{C}$ of $\Omega$, that correspond to the coarse-grained information we can obtain about the physical systems. These 
sets are often called events. The family $\mathcal{C}$ containing the events has to satisfy some natural mathematical conditions: in mathematical terms $\mathcal{C}$ has to be a $\sigma$-field. The relevant conditions are the following

A1. $\Omega \in \mathcal{C}$ : if an experiment is performed one of the outcomes will occur.

A2. $\emptyset \in \mathcal{C}$ : it is impossible that no outcome results if an experiment is performed.

A3. If $A \in \mathcal{C}$, then $\Omega-A \in \mathcal{C}$ : if $A$ is a possible measurement outcome then so can be its complement.

A4. If $A, B \in \mathcal{C}$, then $A \cup B \in \mathcal{C}$ and $A \cap B \in \mathcal{C}$ : unions and intersections of experimental outcomes are also possible experimental outcomes.

A5. For countably many $A_{n} \in \mathcal{C}, n=1,2, \ldots, \cup_{n=1}^{\infty} \in \mathcal{C}$. This is a technical condition particularly relevant when dealing with the case where $\Omega$ is a manifold.

Equipping $\Omega$ with a $\sigma$-field turns it into a measurable space. We shall often focus on a particular set of events $A_{i}$. By repeatedly applying the operations of countable union, intersection and complementation we can construct a $\sigma$-field generated by the collection $A_{i}$. This $\sigma$-field will be denoted as $\sigma\left(A_{i}\right)$.

\section{Some examples}

1. If we take a single set $A$, then $\sigma(A)=\{\emptyset, \Omega, A, \Omega-A\}$.

2. One can also consider the $\sigma$-field that is generated by a set of mutually disjoint subsets $A_{i}$ of $\Omega$, that are also exclusive. This essentially represents to a pointer device, each $i$ a position of the pointer. This field will be denoted as $\sigma(\{A\})$.

3. If $\Omega$ is a topological space, we usually consider the $\sigma$-field generated by all closed subsets of $\Omega$. This is known as the Borel $\sigma$-field $\mathcal{B}(\Omega)$. If we consider the real line $\mathbf{R}$, clearly the corresponding Borel field $\mathcal{B}(\mathbf{R})$ (or $\mathcal{B}$ for short) is generated by all interval $[a, b], a, b \in \mathbf{R}$. We shall mostly assume our $\sigma$-fields to be Borel fields.

4. If we have a function $F: \Omega \rightarrow \mathbf{R}$, we can define the $\sigma$-field generated by $F$ as the one generated of all sets of the form $F^{-1}(B)$, where $B$ is a Borel set on $\mathbf{R}$.

An observable is what is actually determined in an experiment. Since in experiments we eventually come to measure real numbers (or occasionally integers, which can be embedded into the real numbers) the mathematical object that 
would represent the notion of observable is a map from $\Omega$ to $\mathbf{R}$. However, not all possible maps will do: the structure of the $\sigma$-fields has to be preserved. This amounts to the following condition.

If $f: \Omega \rightarrow \mathbf{R}$ and $C \in \mathcal{B}$, then $f^{-1}(C) \in \mathcal{C}$.

Such functions are called measurable and in the language of probability theory are known as random variables. We shall denote the space they belong to in as $F(\Omega)$. In the following, unless it is explicitly specified, any function we will refer to, will be an element of $F(\Omega)$.

Among all functions, important are characteristic functions of the various subsets of $\Omega$. These are defined as

$$
\begin{aligned}
\chi_{A}(x) & =1, x \in \Omega \\
& =0, x \notin \Omega
\end{aligned}
$$

An important property of the characteristic functions is the following. If $\lambda$ is a possible value of a random variable $f$ and $A_{\lambda}=f^{-1}(\lambda)$, then it is evident

$$
f=\int d \lambda \lambda \chi_{A_{\lambda}}
$$

This relationship is the prototype of the most important spectral theorem in the case of Hilbert space observables and its analogue in the commutative case.

\subsection{The decoherence functional}

So far our construction has (deliberately) been identical to that of classical probability theory. Now we come to the point of departure. For classical probability theory this is the point to define a probability measure. Let us for the sake of completeness and later comparisons give its standard definition.

A probability measure $p$ on $\Omega$ is a map $p: \mathcal{A} \rightarrow[0,1]$, such that

$-p(\emptyset)=0,($ null triviality $)$

- $p(\Omega)=1,($ normalisation $)$

- for $A, B \in \mathcal{C}$, such that $A \cap B=\emptyset, p(A \cup B)=p(A)+p(B)$, (additivity).

Note, that the additivity condition is often strengthened to include countable unions of mutually disjoint sets $A$. Clearly this definition implies that $p$ can be extended to any measurable function on $\Omega$

Now in quantum theory (and more particularly histories), one measures relative phases in addition to probabilities. As we explained earlier the object that incorporates them is the decoherence functional. One then needs to give some basic properties or axioms by which a decoherence functional will be defined. This 
has been done by Gell-Mann and Hartle [4] and subsequently generalised by Isham and Linden [5, 31], in the context of consistent histories. Their construction lay within the consistent histories approach. They, therefore, considered more general structures for the field of events. Our scheme here is a adoption of theirs in our present context.

Before we give the axioms for the decoherence functional, we should remark that the symbol $D$ or $d$ canonically used in the literature (and employed in the previous section) is very unwieldy and prone to confusion with differentials. For this reason, we took the liberty to denote the decoherence functional as $\Phi$ (for phase) to denote its most important role as the carrier of the phase information.

A decoherence functional $\Phi$ is a map from $\mathcal{C} \times \mathcal{C} \rightarrow \mathbf{C}$, such that the following conditions are satisfied

B1. Null triviality: For any $A \in \mathcal{C}, \Phi(\emptyset, A)=0$.

In terms of our interpretation of the off-diagonal elements of the decoherence functional as corresponding to Pancharatnam phases, there can be no phase measurement if one of the two beams that have to be interfered is absent.

B2. Hermiticity: For $A, B \in \mathcal{C}, \Phi(B, A)=\Phi^{*}(A, B)$.

Clearly the phase difference between two histories becomes opposite if we exchange the sequence, by which these histories are considered.

B3. Positivity: For any $A \in \mathcal{C}, \Phi(A, A) \geq 0$.

This amounts to the fact that the diagonal elements of the decoherence functional are interpreted as probabilities (albeit non-additive). Operationally probabilities are defined by the number of times a particular event occurred in the ensemble and by definition they can only be positive.

B4. Normalisation: $\Phi(\Omega, \Omega)=1$.

Clearly, if no measurement takes place the intensity of the beam would never change.

B5. Additivity: If $A, B, C \in \mathcal{C}$ and $A \cap B=\emptyset$, then $\Phi(A \cup B, C)=\Phi(A, C)+$ $\Phi(B, C)$.

There is no intuitive operational reason, why this should be the case. This property is equivalent to the superposition principle of quantum theory and we can consider that it is forced upon us by experimental results. Of course, this is 
the property that makes the decoherence functional the natural object to use. Note, that it is probably natural to extend it for countable unions of mutually disjoint subsets $A_{i}$.

B6. Boundedness: For all $A, B \in \mathcal{C},|\Phi(A, B)| \leq 1$.

This is suggested by the measurability of our general discussion in section 2.2In standard quantum theory this condition is satisfied by homogeneous histories 2 , i.e histories that can be written in the form $\left(\alpha_{1}, t_{1} ; \alpha_{2}, t_{2} ; \ldots ; \alpha_{n}, t_{n}\right)$. It is not true for their generic conjunctions, when viewed as propositions. These conjunctions are called inhomogeneous histories in [5, 31]. When the lattice of propositions is Boolean like in our case all conjunctions of homogeneous histories are homogeneous histories and we can expect this condition to be valid.

Now a decoherence functional $\Phi$ can be extended into a functional over $F(\Omega)$. One can clearly define its values on a pair of functions $F, G \in F(\Omega)$ by additivity

$$
\Phi(F, G):=\int d \lambda d \mu \lambda \mu \Phi\left(F^{-1}(\{\lambda\}), G^{-1}(\{\mu\})\right),
$$

where $\lambda$ and $\mu$ are possible real values for the functions $f$ and $g$ respectively.

We keep the same symbol $\Phi$ for the decoherence functional, viewed as functional either on $\mathcal{C}$ or on $F(\Omega)$. The properties 1-6 are then translated as follows:

C1. Null triviality: For any $F \in F(\Omega), \Phi(0, F)=0$.

C2. Hermiticity: For any $F, G \in F(\Omega), \Phi(G, F)=\Phi^{*}(F, G)$.

C3. Positivity: For any $F \in F(\Omega), \Phi(F, F) \geq 0{ }^{3}$.

C4. Normalisation: $\Phi(1,1)=1$.

C5. Additivity: For any $F, G, H \in F(\Omega), \Phi(F, G+H)=\Phi(F, G)+\Phi(F, H)$.

C6. Boundedness: For any $F, G \in F(\Omega),|\Phi(F, G)| \leq\|F\|\|G\|$, where $\|\cdot\|$ denotes the supremum norm on $\Omega$.

Whenever $\Omega$ is a space equipped with a measure $d x$ (of the Lebesque type), we can focus our attention to decoherence functionals that can be obtained by

\footnotetext{
${ }^{2}$ The proof for a decoherence functional of the form (2.2) is straightforward:

$$
|\Phi(\alpha, \beta)|=\left|\operatorname{Tr}\left(\hat{C}_{\alpha} \hat{\rho}_{0} \hat{C}_{\beta}^{\dagger}\right)\right| \leq \operatorname{Tr} \mid\left(\hat { C } _ { \alpha } \hat { \rho } _ { 0 } \hat { C } _ { \beta } ^ { \dagger } \left|\leq \operatorname{Tr} \hat{\rho}_{0}\left\|\hat { C } _ { \alpha } \left|\left\||| \hat{C}_{\beta}^{\dagger}\right\| \leq 1 .\right.\right.\right.\right.
$$
}

\footnotetext{
${ }^{3}$ This property is satisfied in quantum theory, since by additivity we have $\Phi(F, F)=$ $\sum_{\alpha, \beta} \lambda_{\alpha} \lambda_{\beta} \Phi\left(A_{\alpha}, A_{\beta}\right)$, where we wrote the spectral decomposition $F=\sum \lambda_{\alpha} \chi_{A_{\alpha}}$. Using equation (2.2) let us write $\hat{A}=\sum_{\alpha} \lambda_{\alpha} \hat{C}_{\alpha}$, then $\Phi(F, F)=\operatorname{Tr}\left(\hat{\rho}_{0} \hat{A}^{\dagger} \hat{A}\right) \geq 0$. In fact, for complex valued $F$ we have by the same token $\Phi(\bar{F}, F) \geq 0$.
} 
a density: i.e. we can write

$$
\Phi(F, G)=\int F(x) G\left(x^{\prime}\right) \Phi\left(d x, d x^{\prime}\right)=\int d x d x^{\prime} F(x) G\left(x^{\prime}\right) v\left(x, x^{\prime}\right)
$$

in terms of a complex-valued density $v$ on $\Omega \times \Omega$. In a precise mathematical language $v\left(x, x^{\prime}\right)$ is the Radon-Nikodym derivative of $\Phi(\cdot, \cdot)$ with respect to the Lebesque measure $d x \otimes d x$ on $\Omega \times \Omega$. This amounts to viewing $\Phi$ as a complex-valued measure on $\Omega \times \Omega$. This perspective will be very useful in the mathematical development of our theory as we can exploit a number formal similarities with the complex measures.

In particular, we can employ the powerful Radon-Nikodym theorem, which states that if $\nu$ is a $\sigma$-additive measure over a $\sigma$-field over which another $\sigma$ additive measure $\mu$ is define, then up to a set of measure zero we can write a random variable $f$ such that $\nu(A)=\int_{A} f d \mu$. This function $f$ is the RadonNikodym derivative, which was employed earlier to define the density $v$. This theorem forms the basis of the formulation of conditional expectations.

We should also remind the reader that the axioms 1-6 for the decoherence functional are essentially the properties that a density matrix would have if it was viewed as a bilinear kernel over a space $\Omega$. It is in this sense that the decoherence functional is a generalisation of the density matrix in the history setting.

A pair of events for which $\Phi(A, B)=0$ is called a pair of measure zero, while any event $A$ for which $\Phi(A, B)=0$, for all $B$ is called an event of measure zero.

\subsection{Simple examples}

Let us now give some examples by which a decoherence functional appears in quantum theory. Our focus is eventually histories; however, at this point we shall consider systems at a single moment of time, mostly in order to make the connection with the standard formalism of quantum theory more clear. We then seek to see how a decoherence functional of the form $\operatorname{Tr}(\hat{A} \hat{\rho} \hat{B})$ can be represented in terms of commutative observables

Example 1: Let us consider the case that $\Omega=\mathbf{R}$ corresponds to the spectrum of a self-adjoint operator $A$. If $\rho$ is the density matrix of the system then the distribution function for the decoherence functional is

$$
v\left(x, x^{\prime}\right)=\rho\left(x, x^{\prime}\right) \delta\left(x, x^{\prime}\right)
$$

Then for any Borel subsets of $\mathbf{R} A, B$ we get the trivial result (no complex phases)

$$
\Phi(A, B)=\int_{A \cap B} d x \rho(x, x)=p(A \cap B)
$$


Example 2: More interesting is the case where $\Omega$ is taken to be the classical phase space and the correspondence between quantum operators and classical objects is taken through the Wigner transform. Let us consider for simplicity an one dimensional system, characterised by the canonical commutation relation

$$
[q, p]=i
$$

We can write the Weyl operator $\hat{U}(x, \xi)=e^{i x \hat{p}+i \xi \hat{q}}$. The Wigner transform provides a map from any function on the system's Hilbert space and the classical phase space:

$$
\hat{A} \rightarrow F_{\hat{A}}=\int d x d \xi e^{-i q \xi-i p x} \operatorname{Tr}(\hat{A} \hat{U}(x, \xi)):=\operatorname{Tr}(\hat{A} \hat{\Delta}(q, p),
$$

The density corresponding $\rho$ is then

$$
v\left(q, p ; q^{\prime} p^{\prime}\right)=\operatorname{Tr}\left(\hat{\Delta}(q, p) \rho \hat{\Delta}\left(q^{\prime}, p^{\prime}\right)\right)
$$

Using the fact that $\hat{U}(x, \xi) \hat{U}\left(x^{\prime}, \xi^{\prime}\right)=e^{i\left(\xi x^{\prime}-\xi^{\prime} x\right)} U\left(x+x^{\prime}, \xi+\xi^{\prime}\right)$ we derive that

$$
v\left(q, p ; q^{\prime} p^{\prime}\right)=e^{i\left(q p^{\prime}-p q^{\prime}\right)} W\left(q^{\prime}-q, p-p^{\prime}\right),
$$

where $W(q, p)=\operatorname{Tr}(\hat{\rho} \hat{\Delta}(q, p))$ is the Wigner function of the classical system. So if we consider two subsets of the phase space $A$ and $B$ we have

$$
\Phi(A, B)=\int_{A} d q d p \int_{B} d q^{\prime} d p^{\prime} e^{i\left(q p^{\prime}-p q^{\prime}\right)} W\left(q^{\prime}-q, p-p^{\prime}\right)
$$

It is clear that the probability $\Phi(A, A)$ is not given by the Wigner function as a probability distribution. This corresponds to the well known fact that the Wigner function is not a genuine probability distribution, since it is negative. We can get a classical probabilistic description only if we consider that the volume of $A$ and $B$ is much larger than unity (remember $\hbar=1$ ), in which case some smeared (positive) version of the Wigner function becomes a probability distribution. It is worth remarking that if we are able to produce devices that correspond to accurate phase space measurements, then one would be able to determine an interference phase even in a single moment of time.

Example 3: An alternative description of phase space properties is by means of the coherent states. If we define the coherent states as

$$
|x, \xi\rangle=\hat{U}(x, \xi)|0\rangle,
$$


where $|0\rangle$ is a reference state, often taken to be the ground state of the system's Hamiltonian. Using the coherent states to describe the classical phase space we have

$$
v\left(x, \xi ; x^{\prime}, \xi^{\prime}\right)=\left\langle x \xi|\rho| x^{\prime} \xi^{\prime}\right\rangle\left\langle x^{\prime} \xi^{\prime} \mid x \xi\right\rangle
$$

\subsection{Combination of subsystems}

If we have two separate systems, characterised by sample spaces $\Omega_{1}$ and $\Omega_{2}$, then the combined system is characterised by the Cartesian product $\Omega_{1} \times \Omega_{2}$. If also the two systems are independent and characterised by decoherence functionals $\Phi_{1}$ and $\Phi_{2}$ respectively, then the combined system is described by the decoherence functional $\Phi_{1} \otimes \Phi_{2}$, which is defined as

$$
\left(\Phi_{1} \otimes \Phi_{2}\right)\left(A_{1} \times A_{2}, B_{1} \times B_{2}\right)=\Phi_{1}\left(A_{1}, B_{1}\right) \times \Phi_{2}\left(A_{2}, B_{2}\right) .
$$

\subsection{Conditioning}

\subsubsection{The classical case}

Conditioning it is a very important part of classical probability; it is the mathematical implementation of the idea that when we obtain information from an experiment, we need to modify the way we describe the system (i.e. the probability distribution) in order to account for the new information. The prototype of conditioning classically is the notion of conditional probability, i.e. the probability that $A$ will take place when we have verified that $B$ occured. Writing the conditional probability as $p(A \mid B)$ we have that

$$
p(A \mid B)=\frac{p(A \cap B)}{p(B)}
$$

Now this is a very restrictive definition of conditional probability, since it cannot be generalised to the case that $p(B)=0$; objects like propagators are defined through conditional probabilities of events with zero measure.

The most general possible definition that allows such generalisations is that of conditional expectation. This refers to a $\sigma$-field, which is a subfield $\mathcal{A}$ of $\mathcal{C}$. The elements of the subfield $\mathcal{A}$ correspond to the events we want to condition.

In classical probability the conditional expectation of a random variable $F \in F(\Omega)$ with respect to $\mathcal{A}$ is defined as a random variable denoted as $p(F \mid \mathcal{A})$ that is measurable with respect to $\mathcal{C}$ and is such that for all $B \in \mathcal{A}$

$$
\int_{B} p(d x) p(F \mid \mathcal{A})=\int_{B} F(x) p(d x)
$$

The existence of such variables can be proved by virtue of the Radon-Nikodym theorem for any subfield $\mathcal{C}$. It is unique up to a set of measure zero. To 
understand the nature of this object $p(F \mid \mathcal{A})$ consider the case of the subfield corresponding to a pointer device $\sigma(\{A\})$. If $p\left(A_{i}\right) \neq 0$ then we have

$$
p(F \mid \sigma(\{A\}))(x)=\sum_{i} \frac{p\left(F \chi_{A_{i}}\right)}{p\left(A_{i}\right)} \chi_{A_{i}}(x)
$$

However, the definition of $p\left(F \mid \sigma(\{A\})\right.$ makes sense also for cases where $p\left(A_{i}\right)=$ 0 for some values of $i$. One just drops these values from the summation in (3. $19)$.

If $F=\chi(C)$ for some subset $C$ of $\Omega$ we define the conditional probability as a random variable $p(C \mid \mathcal{C})=p\left(\chi_{C} \mid \mathcal{A}\right)$ and in the case of equation (3. 19)

$$
p(C \mid \sigma(\{A\}))(x)=\sum_{i} \frac{p\left(C \cap A_{i}\right)}{p\left(A_{i}\right)} \chi_{A_{i}}(x)
$$

Note that for a fixed value of $x \in A_{i}$, which refers to a definite experimental outcome we have

$$
p\left(C \mid \sigma(\{A\})=\frac{p\left(C \cap A_{i}\right)}{p\left(A_{i}\right)},\right.
$$

which amounts to equation (3. 17) for conditional probabilities. However, it is not always true that we can define a probability distribution out of the fixed choice of the variable $x$.

\subsubsection{The quantum case}

The above-mentioned logic can be straightforwardly generalised for conditioning with respect to the decoherence functional, since this can be viewed as a complex measure on $\Omega \times \Omega$. The different physical meaning of its contents, though, we lead us to distinctions that are absent in the classical case.

First, given a subalgebra $\mathcal{A}$ of $\mathcal{C}$, we can define an object that is mathematically analogous to the conditional expectation. That is, for a pair of random variables $F$ and $G$ we can define a function on $F_{C}(\Omega) \otimes F_{C}(\Omega)$, which we shall write us $\tilde{\Phi}(F, G \mid \mathcal{C})$ and call the conditional pair. This is measurable on $\mathcal{A} \times \mathcal{A}$ and for all $B, B^{\prime} \in \mathcal{A}$

$$
\int_{B \times B^{\prime}} \tilde{\Phi}(F, G \mid \mathcal{A})\left(x, x^{\prime}\right) \Phi\left(d x, d x^{\prime}\right)=\int_{B \times B^{\prime}} F(x) g\left(x^{\prime}\right) \Phi\left(d x, d x^{\prime}\right)
$$

Unlike the classical case $\tilde{\Phi}(F, G \mid \mathcal{A})$ is not a random variable, not even a pair of random variables as it is correlated with respect to the two copies of $\Omega$ entering its arguments. For the case of the pointer sub-field and when $\Phi\left(A_{i}, A_{j}\right) \neq 0$ for all $A_{i}, A_{j}$ it reads

$$
\tilde{\Phi}(F, G \mid \sigma(\{A\}))\left(x, x^{\prime}\right)=\sum_{i j} \frac{\Phi\left(F \chi_{A_{i}}, G \chi_{A_{j}}\right)}{\Phi\left(A_{i}, A_{j}\right)} \chi_{A_{i}}(x) \chi_{A_{j}}\left(x^{\prime}\right)
$$


An important property of the conditional pair is the following:

If $\mathcal{A}^{\prime} \subseteq \mathcal{A}$, then we have that

$$
\tilde{\Phi}\left[\tilde{\Phi}(F, G \mid \mathcal{A}) \mid \mathcal{A}^{\prime}\right]=\tilde{\Phi}\left[\tilde{\Phi}\left(F, G \mid \mathcal{A}^{\prime}\right) \mid \mathcal{A}\right]=\tilde{\Phi}\left(F, G \mid \mathcal{A}^{\prime}\right),
$$

This means that the conditioning with respect to the more stringent field $\mathcal{A}^{\prime}$ prevails. The proof follows from the definition of $\tilde{\Phi}$ and can be intuitively viewed by examining the case of pointer subfields.

Another important property is that normalisation is preserved by conditioning, in the sense that

$$
\tilde{\Phi}(1,1 \mid \mathcal{A})=1
$$

The conditional pair is the correct mathematical way to implement the notion of conditioning for decoherence functionals. It naturally arises from the similarity of $\Phi$ to a complex-valued measure. It has, however, little direct physical meaning as it stands. For instance, taking a fixed value for the arguments $x$ and $x^{\prime}$ does not give a decoherence functional (unlike the classical case), because it does not satisfy the positivity condition.

However, we can concentrate on the diagonal elements of the conditional pair by defining

$$
\Phi(F, G \mid \mathcal{C})=\int d x^{\prime} \delta\left(x, x^{\prime}\right) \tilde{\Phi}(F, G \mid \mathcal{A})\left(x, x^{\prime}\right)
$$

$\Phi(F, G \mid \mathcal{C})$ is a complex valued random variable, which we shall call the conditional correlation of $F$ and $G$. In the case of the pointer subfield we have

$$
\Phi(F, G \mid \sigma(\{A\}))(x)=\sum_{i} \frac{\Phi\left(F \chi_{A_{i}}, G \chi_{A_{i}}\right)}{\Phi\left(A_{i}, A_{i}\right)} \chi_{A_{i}}(x)
$$

For a fixed value of $x \in A_{i}$ (which amounts to a determination of what the pointer actually showed) this gives a conditioned decoherence functional

$$
\frac{\Phi\left(F \chi_{A_{i}}, G \chi_{A_{i}}\right)}{\Phi\left(A_{i}, A_{i}\right)}
$$

If the $A_{i}$ corresponds to a subset of the spectrum of some operator it will be represented in quantum theory by a projection operator $\hat{P}$ (as in example 1) this gives the familiar reduction of the wave packet rule

$$
\hat{\rho} \rightarrow \frac{\hat{P} \hat{\rho} \hat{P}}{\operatorname{Tr}(\hat{\rho} \hat{P})} .
$$

For the case that the $\sigma$-field is generated by a family of functions $Z^{a}, a=$ $1, \ldots n$, the corresponding conditioned pair will be denoted as $\tilde{\Phi}\left(F, G \mid Z^{a}\right)$. This 
is a measurable function on $\Omega \times \Omega$. By construction it is measurable with respect to all sets of the form $\left(Z^{a}\right)^{-1}(B) \times\left(Z^{b}\right)^{-1}\left(B^{\prime}\right)$, for $B, B^{\prime}$ Borel sets in $\mathbf{R}$. It can therefore be written as $h\left(Z, Z^{\prime}\right)$, where $h: \mathbf{R}^{n} \times \mathbf{R}^{n} \rightarrow \mathbf{C}$ is a measurable map. We can then define the quantity

$$
\tilde{\Phi}\left(F, G \mid Z^{a}=z^{a}, Z^{b}=z^{\prime b}\right):=\tilde{\Phi}\left(F, G \mid z^{a}, z^{\prime b}\right):=h\left(z, z^{\prime}\right) .
$$

This object will be of great importance in the discussion of the Markov property.

Note that by the same token one can construct a conditional correlation $\Phi\left(F, G \mid z^{a}\right)$.

\section{Quantum processes}

\subsection{Basic structures}

\subsubsection{Space of histories}

Now we shall consider the case, where the space $\Omega$ of events corresponds to histories. We take $\Omega$ to be a space of paths from the set $T$, where the time parameter lies, to a manifold which corresponds to the fine grained alternatives at a single moment of time. The set $T$ will typically be either a closed interval of the real line or the whole real line. The space $\Gamma$ will be taken as the classical phase space of the system and its points will be denoted usually as $z$. The space of histories $\Omega$ will be taken to consist of maps from $T$ to $\Gamma$, that are at least continuous. Note that $\Omega$ is a subset of $\times_{t} \Gamma_{t}$, where $\Gamma_{t}$ is a copy of $\Gamma$ defined at time $t$. The histories -elements of $\Omega$ - will be denoted as $z(\cdot)$.

The $\sigma$-field on $\Omega$ will be generated by the Borel subsets of $\Gamma_{t}$ for all times $t$. Now if $f$ is a measurable function on $\Gamma$, one can define the one-parameter family of measurable functions $F_{t}$ on $\Omega$ as

$$
F_{t}(z(\cdot))=f(z(t))
$$

A quantum process is defined as a triple $\left(\Omega, \Phi, F_{t}^{a}\right)$, where $\Omega$ is a history space as previously defined, $\Phi$ is a decoherence functional and $F_{t}^{a}$ is family of functions indexed by $t$. Usually for $F_{t}^{a}$ we will consider functions of the form (4. 1), with corresponding $f^{a}$ a set of coordinates on $\Gamma$, or the generators of a group acting transitively on $\Gamma$ (as $\Gamma$ is taken to be a symplectic manifold).

\subsubsection{The decoherence functional}

The first issue that concerns us, is how a decoherence functional can be defined on the path space $\Omega$. In [26] we briefly explained that this can be achieved in a similar fashion as probability measures on path spaces are defined in the theory of stochastic processes. This procedure is as follows: 
Let us consider a subset $\Delta_{n}$ of $T=[0, \tau]$ consisting of $n$-time points $\left\{t_{1}, t_{2}, \ldots, t_{n}\right\}$ and define the space $\Gamma^{\Delta_{n}}=\times_{t \in \Delta_{n}} \Gamma_{t}$. The map $i_{\Delta_{n}}$ induces a (measurable) pullback map from $\Omega$ to $\Gamma^{\Delta_{n}}$ defined as

$$
i_{\Delta_{n} *}[z(\cdot)]=\left(z\left(t_{1}\right), z\left(t_{2}\right), \ldots, z\left(t_{n}\right)\right) .
$$

In its turn, we have a push-forward map $i_{\Delta_{n}}^{*}: F\left(\Gamma^{\Delta_{n}}\right) \rightarrow F(\Omega)$, which reads explicitly as

$$
i_{\Delta_{n}}^{*} f[z(\cdot)]=f\left(z\left(t_{1}\right), z\left(t_{2}\right), \ldots, z\left(t_{n}\right)\right),
$$

where $f$ is a function on $\Gamma^{\Delta_{n}}$. All random variables on $\Omega$ that can be written as a pushforward of the form (4. 3) are called cylinder functions with support on $\Delta_{n}$. They generate a large class of random variables on $\Omega$ through a standard procedure: if $F$ is a cylinder function with support on $\Delta_{n}$, we can define the $L^{1}$ norm

$$
\|F\|=\sum_{i=}^{n}\left|F\left(z\left(t_{i}\right)\right)\right|\left(t_{i}-t_{i-1}\right)
$$

where we take $t_{0}=0$. One can complete the space of cylinder functions with respect to this norm, to get a subset $B(\Omega)$ of $F(\Omega)$, consisting of bounded functions, many of which have interesting properties (e.g. continuity).

Now, if there exists a decoherence functional on $\Omega$, it can be pullbacked on $\Gamma^{\Delta_{n}} \times \Gamma^{\Delta_{m}}$, defining decoherence functionals $\Phi_{\Delta_{n} \times \Delta_{m}}$ by its action on functions $f, g \in F\left(\Gamma^{\Delta_{n}}\right)$

$$
\Phi_{\Delta_{n} \times \Delta_{m}}(f, g)=\Phi\left(i_{\Delta_{n}}^{*} f, i_{\Delta_{m}}^{*} g\right)
$$

Now $\Gamma$ is equipped with a Lebesque measure $d z$ (if it is a symplectic manifold the measure is defined by the symplectic form), with respect to which we can write $\Phi_{\Delta_{n} \times \Delta_{m}}$ in terms of distribution functions

$$
\begin{array}{r}
\Phi_{\Delta_{n} \times \Delta_{m}}\left(\prod_{t_{n}} d z_{t_{n}}, \prod_{t_{n}^{\prime}} d z_{t_{n}^{\prime}}^{\prime}\right)= \\
v_{\Delta_{n} \times \Delta_{m}}\left(z_{t_{1}}, \ldots, z_{t_{n}} ; z_{t_{1}^{\prime}}^{\prime}, \ldots, z_{t_{m}^{\prime}}^{\prime}\right) \prod_{t_{i}} d z_{t_{i}} \prod_{t_{i}^{\prime}} d z_{t_{i}^{\prime}}^{\prime}
\end{array}
$$

This implies that the full information of the decoherence functional's action on cylinder sets (and by continuity on the whole of $B(\Omega)$ ) is contained in a hierarchy of distribution functions

$$
v^{n, m}\left(z_{1}, t_{1} ; z_{2}, t_{2} ; \ldots ; z_{n}, t_{n} \mid z_{1}^{\prime}, t_{1}^{\prime} ; z_{2}^{\prime}, t_{2}^{\prime} ; \ldots ; z_{m}^{\prime}, t_{m}^{\prime}\right),
$$

for all integer values of $n$ and $m$. Note that $v_{n, m}$ is here viewed as a function on $\Gamma^{n+m} \times T^{n+m}$. These functions are time-ordered in the sense that their temporal arguments satisfy $t_{1}<t_{2}<\ldots<t_{n}$ and $t_{1}^{\prime}<t_{2}^{\prime}<\ldots<t_{m}^{\prime}$.

Conversely, if we are provided with such a hierarchy of functions, we can define a decoherence functional on $\Omega$, provided some conditions are valid. 
First, for each choice of values of $\Delta_{n}=\left\{t_{1}, \ldots, t_{n}\right\}$ and $\Delta_{m}=\left\{t_{1}^{\prime}, \ldots, t_{m}^{\prime}\right\}$, the corresponding functions on $\Gamma^{\Delta_{n}} \times \Gamma^{\Delta_{m}}$ must be such a to define a decoherence functional satisfying properties C1-C6.

Second, there needs to be a compatibility condition between the decoherence functionals in $\Delta_{n} \times \Delta_{m}$ and $\Delta_{n}^{\prime} \times \Delta_{m}^{\prime}$, if $\Delta_{n} \subseteq \Delta_{n}^{\prime}$ and $\Delta_{m} \subseteq \Delta_{m}^{\prime}$, if both objects are to correspond to the same decoherence functional on $\Omega \times \Omega$. For this reason we demand the

(Kolmogorov) Additivity condition:

$\int d z_{n+1} v^{n+1, m}\left(\left(z_{1}, t_{1} ; z_{2}, t_{2} ; \ldots ; z_{n}, t_{n} ; z_{n+1}, t_{n+1} \mid z_{1}^{\prime}, t_{1}^{\prime} ; z_{2}^{\prime}, t_{2}^{\prime} ; \ldots ; z_{m}^{\prime}, t_{m}^{\prime}\right)=\right.$ $v^{n, m}\left(z_{1}, t_{1} ; z_{2}, t_{2} ; \ldots ; z_{n}, t_{n} \mid z_{1}^{\prime}, t_{1}^{\prime} ; z_{2}^{\prime}, t_{2}^{\prime} ; \ldots ; z_{m}^{\prime}, t_{m}^{\prime}\right)$.

The satisfaction of these conditions guarantees the existence of a well-behaved decoherence functional $\Phi$ on $B(H)$, such that its pull-backs on $\Gamma^{\Delta_{n}} \times \Gamma^{\Delta_{m}}$ are given by the functions of the hierarchy. ( $\Phi$ is said to be the inductive limit of this hierarchy.)

Similar hierarchies have been previously identified in the context of the phase space picture of quantum mechanics: in reference [33] such hierarchies are said to correspond to a generalisation of stochastic processes, while in reference [34] they are employed to prove that the quantum mechanical predictions cannot be recovered by any classical stochastic process.

There are other hierarchies of functions that can be used to define the decoherence functional. For instance, given the ordered distribution functions $v^{n, m}$, we can write the object

$$
\begin{array}{r}
\theta\left(t_{n}-t_{n-1}\right) \ldots \theta\left(t_{2}-t_{1}\right) \theta\left(t_{m}^{\prime}-t_{m-1}^{\prime}\right) \ldots \theta\left(t_{2}^{\prime}-t_{1}^{\prime}\right) \\
v^{n, m}\left(z_{1}, t_{1} ; \ldots ; z_{i}, t_{i} ; \ldots ; z_{j}, t_{j} ; \ldots z_{n}, t_{n} \mid z_{1}^{\prime}, t_{1}^{\prime} ; \ldots ; z_{m}^{\prime}, t_{m}^{\prime}\right)
\end{array}
$$

By fully symmetrising this object separately with respect to its $z_{i}, t_{i}$ and $z_{i}^{\prime}, t_{i}^{\prime}$ entries, we obtain the time-symmetric distribution functions $w^{n, m}$. One can obtain the decoherence functional from such a hierarchy, only of course now one has to demand the additional

Symmetry postulate:

$w^{n, m}\left(z_{1}, t_{1} ; \ldots ; z_{i}, t_{i} ; \ldots ; z_{j}, t_{j} ; \ldots z_{n}, t_{n} \mid z_{1}^{\prime}, t_{1}^{\prime} ; \ldots ; z_{m}^{\prime}, t_{m}^{\prime}\right)=$ $w^{n, m}\left(z_{1}, t_{1} ; \ldots ; z_{j}, t_{j} ; \ldots ; z_{i}, t_{i} ; \ldots z_{n}, t_{n} \mid z_{1}^{\prime}, t_{1}^{\prime} ; \ldots ; z_{m}^{\prime}, t_{m}^{\prime}\right)$.

We should also remark that the requirement of Kolmogorov additivity means that we do not need to specify the full hierarchy $v^{n, m}$ to determine the decoherence functional. It suffices to specify the diagonal distribution functions $v^{N, N}$ for the values of time such that $t_{i}=t_{i}^{\prime}$, i.e. we just need provide

$$
v^{N, N}\left(z_{1}, t_{1} ; z_{2}, t_{2} ; \ldots ; z_{N}, t_{N} \mid z_{1}^{\prime}, t_{1} ; z_{2}^{\prime}, t_{2} ; \ldots ; z_{N}^{\prime}, t_{N}\right)
$$

Every distribution $v^{n, m}$ can be obtained from the $v^{N, N}$ for $N=n+m$. One chooses $n$ values of the time label (for simplicity take $t_{1} \ldots t_{N}$ ) as corresponding 
to the forward in time entries and the remaining $m$ as corresponding to the backward in time ones (for simplicity take $t_{n+1}, \ldots t_{n+m}$ ). Then integrate out over $z_{1}^{\prime}, \ldots z_{n}^{\prime}$ and $z_{n+1} \ldots z_{n}$ to get the hierarchy $v^{n, m}$.

Summarising this section we can say that one can define a decoherence functional on $\Omega$, by specifying a hierarchy of distribution functions (4. 6). We can, therefore, study continuous-time objects, while working with discrete-time expressions.

\subsubsection{Correlation functions}

Part of the definition of stochastic processes is the specification of a family of functions $F_{t}^{i}$. Recalling the discussion in section 2.3, we can see that one can readily define the mixed correlation functions $G^{n, m}$ as

$$
\begin{array}{r}
G^{n, m}\left(a_{1}, t_{1} ; a_{2}, t_{2} ; \ldots ; a_{n}, t_{n} \mid b_{1}, t_{1}^{\prime} ; b_{2}, t_{2}^{\prime} ; \ldots ; b_{m}, t_{m}^{\prime}\right)= \\
\Phi\left(F_{t_{1}}^{a_{1}} F_{t_{2}}^{a_{2}} \ldots F_{t_{n}}^{a_{n}}, F_{t_{1}^{\prime}}^{b_{1}} F_{t_{2}^{\prime}}^{b_{2}} \ldots F_{t_{m}^{\prime}}^{b_{m}}\right)
\end{array}
$$

Note that each correlation function $G^{n, m}$ (for fixed values of $n$ and $m$ ) needs only the information contained in $v^{n, m}$ of equation (4. 6) in order to be determined.

As we discussed in section 2. $G^{n, 0}$ are the time-ordered correlation functions, $G^{0, m}$ the anti-time-ordered correlation functions. In general there are certain relations between functions with the same value of $n+m$. For instance

$$
G^{2,0}\left(a_{1}, t_{1} ; a_{2}, t_{2}\right)=\theta\left(t_{2}-t_{1}\right) G^{1,1}\left(a_{1}, t_{1} \mid a_{2}, t_{2}\right)+\theta\left(t_{1}-t_{2}\right) G^{1,1}\left(a_{2}, t_{2} \mid a_{1}, t_{1}\right)
$$

Once we have the hierarchy $G_{n, m}$ of correlation functions associated to $F_{t}^{a}$ we can define the corresponding closed-time-path generating functional $Z_{F}\left[J_{+}, J_{-}\right]$, which is written in terms of the sources $J_{+}^{a}(t), J_{-}^{a}(t)$ as

$$
\begin{aligned}
& Z_{F}\left[J_{+}, J_{-}\right]=\sum_{n=0}^{\infty} \sum_{m=0}^{\infty} \frac{i^{n}(-i)^{m}}{n ! m !} \\
& \times \sum_{a_{1}, \ldots a_{n}} \sum_{b_{1}, \ldots, b_{m}} \int d t_{1} \ldots d t_{n} d t_{1}^{\prime} \ldots d t_{m}^{\prime} \\
& \times G^{n, m}\left(a_{1}, t_{1} ; \ldots ; a_{n}, t_{n} \mid b_{1}, t_{1}^{\prime} ; \ldots ; b_{m}, t_{m}^{\prime}\right) \\
& \quad \times J_{+}^{a_{1}}\left(t_{1}\right) \ldots J_{+}^{a_{n}}\left(t_{n}\right) J_{-}^{b_{1}}\left(t_{1}^{\prime}\right) \ldots J_{-}^{b_{m}}\left(t_{m}^{\prime}\right)
\end{aligned}
$$

Clearly the CTP generating functional can be written as

$$
Z_{F}\left[J_{+}, J_{-}\right]=\Phi\left(e^{i F \cdot J_{+}}, e^{-i F \cdot J_{-}}\right)
$$

where $F \cdot J_{ \pm}:=\int d t \sum_{i} F_{t}^{i} J_{ \pm}(t)$. From this equation it is easy to see the two main properties that $Z_{F}\left[J_{+}, J_{-}\right]$inherits from the decoherence functional: 
1. Hermiticity: $Z_{F}\left[J_{-}, J_{+}\right]=Z_{F}^{*}\left[J_{+}, J_{-}\right]$.

2. Normalisation: $Z_{F}[0,0]=1$.

In an inverse way one can obtain the correlation functions from $Z\left[J_{+}, J_{-}\right]$by functional differentiation

$$
\begin{aligned}
& G_{F}^{n, m}\left(a_{1}, t_{1} ; \ldots ; a_{n}, t_{n} \mid b_{1} t_{1}^{\prime} ; \ldots, b_{m}, t_{m}^{\prime}\right)= \\
& \left.(-i)^{n} i^{m} \frac{\delta^{n}}{\delta J_{+}^{a_{1}}\left(t_{1}\right) \ldots \delta J_{+}^{a_{n}}\left(t_{n}\right)} \frac{\delta^{m}}{\delta J_{-}^{b_{1}}\left(t_{1}\right) \ldots \delta J_{-}^{b_{m}}\left(t_{m}\right)} Z\left[J_{+}, J_{-}\right]\right|_{J_{+}=J_{-}=0} \cdot(4
\end{aligned}
$$

If the family of functions $F_{t}^{a}$ separates $\Omega$, then the information in $Z_{F}$ is sufficient to reconstruct the whole decoherence functional. Such is the case, for instance, when the $F_{t}^{a}$ are functions of type (4. 1), with $f^{a}$ being a set of coordinates on $\Gamma$.

Gaussian processes. As in classical probability theory of importance are the Gaussian processes; these are processes, whose CTP generating functional is a Gaussian function of the currents. In Gaussian processes all correlation functions are determined by the two-point functions. Namely, if we define

$$
\begin{array}{r}
F^{a}(t)=G^{1,0}(a, t)=G^{0,1}(a, t) \\
i \Delta^{a b}\left(t, t^{\prime}\right)=G^{2,0}\left(a, t ; b, t^{\prime}\right)-F^{a}(t) F^{b}\left(t^{\prime}\right) \\
i K^{a b}\left(t, t^{\prime}\right)=G^{1,1}\left(a, t \mid b, t^{\prime}\right)+G^{1,1}\left(b, t^{\prime} \mid a, t\right)-2 F^{a}(t) F^{b}\left(t^{\prime}\right),
\end{array}
$$

the CTP generating functional for a Gaussian process reads

$$
\begin{array}{r}
Z\left[J_{+}, J_{-}\right]=\exp \left(-\frac{i}{2} J_{+} \cdot \Delta \cdot J_{+}+\frac{i}{2} J_{-} \cdot \bar{\Delta} \cdot J_{-}\right. \\
\left.+i J_{+} \cdot K \cdot J_{-}+i\left(J_{+}-J_{-}\right) \cdot X\right)
\end{array}
$$

Here we wrote $J \cdot \Delta \cdot J^{\prime}=\int d t d t^{\prime} J^{a}(t) \Delta^{a b}\left(t, t^{\prime}\right) J^{b}\left(t^{\prime}\right)$ and the bar is used to denote complex conjugation.

\subsection{The kinematical process}

So far our discussion of quantum processes was rather formal, that is we did not attempt to write down processes that reproduce the quantum mechanical formalism.

In this section, we shall deal with a class of processes that is of greatest importance for the implementation of our programme: the kinematical processes, 
i.e. quantum processes for systems that have vanishing quantum mechanical Hamiltonian. Unlike classical probability theory, where the absence of dynamics makes a stochastic process trivial, in quantum processes the full wealth of quantum mechanical behaviour is manifested already at the kinematical level. In fact, as we shall see the contribution of dynamics is insignificant (with respect to the defining features of quantum processes) compared to the kinematics.

This remark goes back to Heisenberg [35], who actually started his investigations of quantum theory by postulating that the difference between classical and quantum theory is to be found at the kinematical level, in the nature of the basic observables. In the context of histories, Savvidou [17] has uncovered a sharp distinction between kinematics and dynamics; there generically exist different groups implementing kinematical and dynamical transformations and in history theories they coexist. This duality between kinematics and dynamics corresponds to different ways times is manifested in quantum theory as "reduction of the wave packet" and Heisenberg dynamics respectively [17]. This remarkable property of histories is also present in the distinction between geometric and dynamical phase [11]. These facts have enabled us to argue in [11], that all defining properties of quantum theory are found at solely the kinematical level, dynamics being structurally identical with classical ones. In this paper, we shall make this claim more concrete.

\subsubsection{Coherent states}

In our previous work $[12,26]$, when we wanted to discuss the definition of a process in phase space that mimic quantum phenomena, we instictively assumed that the natural way to do so was through the use of the Wigner transform. In other words, we obtained the functions $v_{n, m}$, that define the decoherence functional on phase space by Wigner-transforming the corresponding objects for the Hilbert space decoherence functional. One can then obtain a quantum process on the phase space, through the construction outlined in section 4.2. The expressions for $v_{n, m}$ thus obtained were rather unwieldy and were not useful in proceeding further.

We found that there is a dramatic simplification if we define the phase space distribution functions by means of the coherent states. Recall, that the coherent states are, in general, associated with a canonical group, that is a group $G$ that has an irreducible representation on the Hilbert space $H$, by unitary operators $\hat{U}(g)[36,37]$. Taking a reference normalised vector $|0\rangle$ (often a vacuum state) we can define the Hilbert space vectors $\hat{U}(g)|0\rangle$. Now we define the equivalence relation

$g \sim g^{\prime}$ if $\hat{U}(g)|0\rangle=e^{i \phi} \hat{U}\left(g^{\prime}\right)|0\rangle$, for some phase $e^{i \phi}$.

The quotient space $\Gamma=G / \sim$ is the parameter space labeling the coherent states $|z\rangle, z \in \Gamma$. $\Gamma$ has a rich structure, since the coherent states define an 
injection map $i$ from $\Gamma$ to the projective Hilbert space $P H$. The latter has a Riemannian metric $d s^{2}$ (coming from the real part of the Hilbert space's inner product), a symplectic form $\omega$ coming from the imaginary part of the inner product, the Hopf bundle (which we discussed in section 2.2) and a $U(1)$ connection (the Berry connection $A$ ), such that $\omega=d A$. All these structures can be pullbacked through $i$ on $\Gamma$ making it a symplectic manifold, with a $U(1)$ bundle, a $U(1)$ connection one-form $A$ and a Riemannian metric. Explicitly, these structures read

$$
\begin{aligned}
d s^{2}(\Gamma) & =\| d|z\rangle \|^{2}-|\langle z|d| z\rangle|^{2} \\
A & =-i\langle z|d| z\rangle \\
\omega & =d A
\end{aligned}
$$

where $d$ is the exterior derivative operator on $\Gamma$.

Also $\Gamma$ is a homogeneous space as the group $G$ acts transitively on $\Gamma$ through the map $|z\rangle \rightarrow \hat{U}(g)|z\rangle$.

\subsubsection{Defining the kinematical process}

Let us consider a Hilbert space $H$ carrying a representation $\hat{U}(g)$ of the group $G$, and a choice of reference vector $|0\rangle$ leading to a family of coherent states $|z\rangle, z \in \Gamma$. If we denote by $\hat{A}^{a}$ the self-adjoint operators that generate the group elements, we can define the basic functions $f^{a}$ on $\Gamma$ through

$$
\hat{A}^{a}=\int d z f^{a}(z)|z\rangle\langle z| .
$$

The functions $f^{a}$ are known as the $P$-symbols of $\hat{A}^{a}$ and we implicitly restrict to operators, whose $P$-symbols are measurable functions.

Now, having $\Gamma$ one can define the space $\Omega$ of continuous paths on $\Gamma$ and the family of functions $F_{t}^{a}$ associated to the $f^{a}$ of equation (4. 1). All is missing from the definition of a quantum processes is the specification of a decoherence functional. This is achieved by specifying the hierarchy of ordered distribution functions $v^{n, m}$. To do so, we write the time instants in terms of their ordering $t_{1} \leq t_{2} \leq \ldots t_{n}$, and $t_{1}^{\prime} \leq t_{2}^{\prime} \leq \ldots \leq t_{m}^{\prime}$. If we write $\hat{\alpha}_{z}=|z\rangle\langle z|$ we will have

$$
\begin{array}{r}
v_{z_{0}}^{n, m}\left(z_{1}, t_{1} ; z_{2}, t_{2} ; \ldots ; z_{n}, t_{n} \mid z_{1}^{\prime}, t_{1}^{\prime} ; z_{2}^{\prime}, t_{2}^{\prime} ; \ldots ; z_{m}^{\prime}, t_{m}^{\prime}\right)= \\
\operatorname{Tr}\left(\hat{\alpha}_{z_{n}} \hat{\alpha}_{z_{n-1}} \ldots \hat{\alpha}_{z_{2}} \hat{\alpha}_{z_{1}} \hat{\alpha}_{z_{0}} \hat{\alpha}_{z_{1}^{\prime}} \ldots \hat{\alpha}_{z_{m-1}^{\prime}} \hat{\alpha}_{z_{m}^{\prime}}\right)= \\
\left\langle z_{m}^{\prime} \mid z_{n}\right\rangle\left\langle z_{n} \mid z_{n-1}\right\rangle \ldots\left\langle z_{2} \mid z_{1}\right\rangle\left\langle z_{1} \mid z_{0}\right\rangle\left\langle z_{0} \mid z_{1}^{\prime}\right\rangle\left\langle z_{1}^{\prime} \mid z_{2}^{\prime}\right\rangle \ldots\left\langle z_{m-1}^{\prime} \mid z_{m}^{\prime}\right\rangle .
\end{array}
$$

This process is defined as starting from $z_{0} \in \Gamma$ at $t=0$. One can define more general processes by smearing through $z_{0}$ with a distribution function positive $\rho\left(z_{0}\right)$

$$
v_{\rho}^{n, m}=\int d z_{0} \rho\left(z_{0}\right) v_{z_{0}}^{n, m}
$$


In the Hilbert space language, this amounts to an initial density matrix

$$
\hat{\rho}=\int d z \rho(z)|z\rangle\langle z|
$$

Let us note some distinguishing features of the kinematic process:

1. The expression for the distribution function factorises in products of the form $\left\langle z \mid z^{\prime}\right\rangle$. The knowledge of this inner product, suffices to fully determine the kinematical process. In fact, the distribution function $v^{n, m}$ is known as the $n+m+1$ Bargmann invariant [38].

2. The distributions $v^{n, m}$ do not depend on the values of time $t$, only on their ordering. The same is true for $t^{\prime}$. More than that, if we consider the following cyclic ordering for the time instants $t_{0} \rightarrow t_{1} \rightarrow t_{2} \rightarrow \ldots \rightarrow t_{n} \rightarrow t_{m}^{\prime} \rightarrow \ldots \rightarrow$ $t_{2}^{\prime} \rightarrow t_{1}^{\prime} \rightarrow t_{0}$, the distributions are invariant if we consider any time as origin and then proceed cyclically along the arrows. In other words, the kinematic process manifests the symmetry of a closed time path.

3. Let us consider that the process being defined in the time interval $[0, \tau]$ and consider the distribution function $v^{n, m}$ for large values of $n$ and $m$. Take for simplicity $n=m=N$. Choose also the time instants such that $\left|t_{i}-t_{i-1}\right| \leq$ $\delta t=\tau / N$ for all $i$ and similarly for $t^{\prime}$. Also, let $t_{n}=t_{m}^{\prime}=\tau$. Then we have a discretised approximation to a decoherence functional for continuous paths $z(\cdot), z^{\prime}(\cdot)$, which for $N \rightarrow \infty$ would converge to

$$
\Phi\left(z(\cdot), z^{\prime}(\cdot)\right)=e^{-i \int_{C}\langle z|d| z\rangle}+O\left(\delta t^{2}\right)=e^{i \int_{C} A}+O\left(\delta t^{2}\right),
$$

where $C$ is the closed path obtained by appending the path $z^{\prime}(\cdot)$ with reverse orientation at the end of $z(\cdot)$. The distribution function for the decoherence functional then converges at the large $N$ limit to the holonomy of the pull-back of the Berry connection on $\Gamma$. Of course, this convergence is to be interpreted with a grain of salt as the support of the decoherence functional is on cylinder functions, rather than differentiable ones, for which the holonomy is rigorously defined.

\subsubsection{The one-dimensional particle process}

Let us study the simplest example of a kinematical process, that of a particle at a line. The functions determining the quantum process are the position $x_{t}$ and the momentum $p_{t}$. Let us take for simplicity that the coherent states are Gaussian (the overlap $\left\langle z \mid z^{\prime}\right\rangle$ is Gaussian as in the standard case) and that $z_{0}=0$. (The 
initial point does not really matter as $\Gamma$ is a homogeneous space and there is no dynamics to differentiate between points). We have the following correlation functions

$$
\begin{array}{r}
G^{2,0}\left(x, t ; x^{\prime}, t^{\prime}\right)=G^{0,2}\left(x, t ; x^{\prime}, t^{\prime}\right)=G^{1,1}\left(x, t \mid x^{\prime}, t^{\prime}\right)=\sigma_{x}^{2}(4.27) \\
G^{0,2}\left(p, t ; p^{\prime}, t^{\prime}\right)=G^{0,2}\left(p, t ; p^{\prime}, t^{\prime}\right)=G^{1,1}\left(p, t \mid p^{\prime}, t^{\prime}\right)=\sigma_{p}^{2}(4.28) \\
G^{2,0}\left(x, t ; p, t^{\prime}\right)=G^{0,2}\left(p, t ; x, t^{\prime}\right)=\frac{1}{2}\left[\theta\left(t-t^{\prime}\right)(C+i)+\theta\left(t^{\prime}-t\right)(C-i)\right](4.29) \\
G^{2,0}\left(p, t ; x, t^{\prime}\right)=G^{0,2}\left(x, t ; p, t^{\prime}\right)=\frac{1}{2}\left[\theta\left(t^{\prime}-t\right)(C+i)+\theta\left(t-t^{\prime}\right)(C-i)\right](4.30) \\
G^{1,1}\left(x, t \mid p, t^{\prime}\right)=\frac{1}{2}(C-i)=\bar{G}^{1,1}\left(p, t \mid x, t^{\prime}\right),(4.31)
\end{array}
$$

in terms of $\sigma_{x}^{2}=\left\langle 0\left|\hat{x}^{2}\right| 0\right\rangle, \sigma_{p}^{2}=\left\langle 0\left|\hat{p}^{2}\right| 0\right\rangle$ and $C=\langle 0|\hat{x} \hat{p}+\hat{p} \hat{x}| 0\rangle$.

The corresponding CTP generating functional is of the form (4.18) with kernels

$$
\begin{aligned}
i \Delta\left(t, t^{\prime}\right) & =\left(\begin{array}{cc}
\sigma_{x}^{2} & \frac{1}{2}\left[C+i \eta\left(t-t^{\prime}\right)\right] \\
\frac{1}{2}\left[C-i \eta\left(t-t^{\prime}\right)\right] & \sigma_{p}^{2}
\end{array}\right) \\
i K\left(t, t^{\prime}\right) & =\left(\begin{array}{cc}
\sigma_{x}^{2} & \frac{1}{2}(C-i) \\
\frac{1}{2}(C+i) & \sigma_{p}^{2}
\end{array}\right),
\end{aligned}
$$

where $\eta\left(t-t^{\prime}\right)=\theta\left(t-t^{\prime}\right)-\theta\left(t^{\prime}-t\right)$.

The fact to note is that it is the correlation between position and momentum that causes the CTP generating functional to be complex. In absence of this the kinematical process would have a completely real-valued generating functional.

\subsection{Introducing dynamics}

The standard way to introduce dynamics is by the introduction of a Hamiltonian operator. Its effect is a change and the introduction of an explicit time dependence in the kernels $\left\langle z \mid z^{\prime}\right\rangle$. In other words, the definition proceeds similarly as the kinematical process, only now the distributions $v^{n, m}$ read

$$
\begin{array}{r}
v_{z_{0}}^{n, m}\left(z_{1}, t_{1} ; z_{2}, t_{2} ; \ldots ; z_{n}, t_{n} \mid z_{1}^{\prime}, t_{1}^{\prime} ; z_{2}^{\prime}, t_{2}^{\prime} ; \ldots ; z_{m}^{\prime}, t_{m}^{\prime}\right)= \\
\left\langle z_{m}^{\prime}\left|e^{-i \hat{H}\left(t_{m}^{\prime}-t_{n}\right)}\right| z_{n}\right\rangle\left\langle z_{n}\left|e^{-i \hat{H}\left(t_{n}-t_{n-1}\right)}\right| z_{n-1}\right\rangle \ldots \\
\times\left\langle z_{2}\left|e^{-i \hat{H}\left(t_{2}-t_{1}\right)}\right| z_{1}\right\rangle\left\langle z_{1}\left|e^{-i \hat{H}\left(t_{1}-t_{0}\right)}\right| z_{0}\right\rangle \\
\times\left\langle z_{0}\left|e^{i \hat{H}\left(t_{1}^{\prime}-t_{0}\right)}\right| z_{1}^{\prime}\right\rangle\left\langle z_{1}^{\prime}\left|e^{i \hat{H}\left(t_{2}^{\prime}-t_{1}^{\prime}\right)}\right| z_{2}^{\prime}\right\rangle \ldots\left\langle z_{n-1}^{\prime}\left|e^{i \hat{H}\left(t_{n}^{\prime}-t_{n-1}^{\prime}\right)}\right| z_{n}^{\prime}\right\rangle .
\end{array}
$$

We see then that the basic object by which the decoherence functional is constructed is the "coherent state propagator" $\chi\left(z, z^{\prime} \mid t\right):=\left\langle z\left|e^{-i \hat{H} t}\right| z^{\prime}\right\rangle$.

Now, let us consider the following. Consider a process with respect to the kinematical decoherence functional (which we shall denote as $\Phi_{0}$ ), but which is 
defined with respect to another set of variables ${ }^{H} F_{t}^{a}$, defined as

$$
\hat{A}^{a}(t)=\int d z^{H} F_{t}^{a}[z(\cdot)]|z(t)\rangle\langle z(t)|
$$

in terms of the Heisenberg picture operators

$$
\hat{A}^{a}(t)=e^{i \hat{H}\left(t-t_{0}\right)} \hat{A}^{a} e^{-i \hat{H}\left(t-t_{0}\right)} .
$$

Note that the construction of these objects employ the time label $t$ in two distinct fashions, one kinematical as the argument of the coherent state and one dynamical in the exponentiation of the Hamiltonian.

The important point is that the process $\left(\Omega, \Phi_{H}, F_{t}^{a}\right)$ is completely isomorphic to the process $\left(\Omega, \Phi_{0},{ }^{H} F_{t}^{a}\right)$ as they have completely identical correlation functions.

The physical interpretation of this fact has been given by Savvidou [17]. Let us define $X_{t}^{a}(s)$ by

$$
e^{i \hat{H} s} \hat{A}^{a} e^{-i \hat{H} s}=\int d z_{t} X_{t}^{a}(s)\left|z_{t}\right\rangle\left\langle z_{t} \mid z(t)\right\rangle .
$$

It is true that $X_{t}^{a}\left(s=t-t_{0}\right)={ }^{H} F_{t} . X_{t}^{a}(s)$ can be obtained from $F_{t}^{a}$ by the solution of a deterministic set of equations. To see this note that for small $s$

$$
e^{i \hat{H} s} \hat{A}^{a} e^{-i \hat{H} s} \simeq A^{a}+i s \int d z_{t} X_{t}^{a}\left(z_{t}\right)\left[\hat{H}, \hat{P}_{z_{t}}\right]
$$

where $\hat{P}_{z}=|z\rangle\langle z|$. If we define the kernel $\alpha\left(z, z^{\prime}, z^{\prime \prime}\right)$ by

$$
\left[\hat{P}_{z}, \hat{P}_{z}^{\prime}\right]=\int d z^{\prime \prime} \alpha\left(z, z^{\prime}, z^{\prime \prime}\right) \hat{P}_{z^{\prime \prime}}
$$

and write $\hat{H}=\int d z h(z) \hat{P}_{z}$, in terms of the $P$-symbol $h$ of the Hamiltonian, it is easy to verify that

$$
\frac{d}{d s} X_{t}^{a}(s)=V_{H}\left(X_{t}^{a}\right)
$$

where $V_{H}$ is an operator on functions on $\Gamma$ givn by

$$
V_{H}(f)(z)=\int d z^{\prime} \beta\left(z, z^{\prime}\right) f\left(z^{\prime}\right),
$$

in terms of $\beta\left(z, z^{\prime}\right)=\int z^{\prime \prime} \alpha\left(z^{\prime}, z^{\prime \prime}, z\right) h\left(z^{\prime \prime}\right)$.

The initial conditions fpr these equations are $X_{t}^{a}\left(s=t_{0}\right)=F_{t}^{a}$. This means that the process $\left(\Omega, \Phi_{H}, F_{t}^{a}\right)$ is obtained by first evolving the $F_{t}^{a}$ according to deterministic equations of motion - separately for each $t$ - and then constructing the correlation functions, when the initial conditions are distributed according to the kinematic process. 


\subsection{Quantum differential equations}

We can make the statement about the overriding importance of kinematics more precise, by introducing the notion of a quantum differential equation. This is meant to be the analogue of a stochastic differential equation in classical probability theory.

Such equations arise when one seeks to understand, how single time observables, i.e. functions on $\Gamma$ change in time. Let us consider an observable $f^{a}$ that

has some fixed value at time $t$ as the system is in the state $z(t)$. In fact, we shall have that

$$
f^{a}(z(t))=F_{t}^{a}[z(\cdot)]
$$

for the process $F^{a}$. Let us consider the description of the system in terms of the process $\left(\Omega, \Phi_{0},{ }^{H} F_{t}^{a}\right)$. At the next moment $t+\epsilon, f^{a}$ will go the function $\tilde{f}^{a}=f^{a}+\epsilon V_{H}\left(f^{a}\right)+O\left(\epsilon^{2}\right)$, which is however a function on $\Gamma_{t+\epsilon}$. This implies that

$$
\begin{array}{r}
\left.\delta f^{a}(z(t))\right):=\tilde{f}^{a}(z(t+\epsilon))-f^{a}(z(t)) \\
=f^{a}(z(t+\epsilon))-f^{a}(z(t))+\epsilon V_{H}\left(f^{a}\right)(z(t))+O\left(\epsilon^{2}\right) \\
=\left(F_{t+\epsilon}^{a}-F_{t}^{a}\right)+\epsilon V_{H}\left(f^{a}\right)(z(t))
\end{array}
$$

Taking $\epsilon=\delta t$ we have the formal equation

$$
d f^{a}(t)-V_{H}\left(f^{a}(t)\right)=d F_{t}^{a},
$$

which can also be written as

$$
\frac{d f^{a}}{d t}-V_{H}\left(f^{a}(t)\right)=\dot{F}_{t}^{a}
$$

This equation is the quantum analogue of a stochastic differential equation. It can be interpreted as referring to an individual quantum system and stating that the rate of change of any function $f$ equals a deterministic part plus a random "external force", which is distributed over the ensemble according to the kinematical process of the system. As we argued earlier, quantum theory has its origins in the kinematical process.

The particle at a line Let us now consider the correlation functions $\dot{F}_{t}^{a}$ for the particle in one dimension. It is easy to check that the expectation values for $\dot{x}$ and $\dot{p}$ vanish in the kinematical process and so do all two-point correlation functions that contain a pair of $\dot{x}$ and $\dot{p}$ 's. We have however,

$$
\begin{array}{r}
G^{2,0}\left(\dot{x}, t ; \dot{p}, t^{\prime}\right)=G^{0,2}\left(\dot{p}, t ; \dot{x}, t^{\prime}\right)=-\frac{i}{2} \partial_{t}^{2} \eta\left(t-t^{\prime}\right) \\
G^{2,0}\left(\dot{p}, t ; \dot{x}, t^{\prime}\right)=G^{0,2}\left(\dot{x}, t ; \dot{p}, t^{\prime}\right)=\frac{i}{2} \partial_{t}^{2} \eta\left(t-t^{\prime}\right) \\
G^{1,1}\left(x, t \mid p, t^{\prime}\right)=G^{1,1}\left(p, t \mid x, t^{\prime}\right)=0 .
\end{array}
$$


By $\dot{x}$ we denote $\frac{1}{\epsilon}\left(x_{t+\epsilon}-x_{t}\right)$ for positive $\epsilon \rightarrow 0$ and similarly for $\dot{p}$. While $\partial_{t}^{2} \eta\left(t-t^{\prime}\right)$ denotes the limit of $\eta\left(t-t^{\prime}\right)+\eta\left(t-t^{\prime}-\epsilon\right)-2 \eta\left(t-t^{\prime}\right)$, in terms of $\eta\left(t-t^{\prime}\right)$. This is a version of the first derivative of the $\delta$-function.

The corresponding CTP generating functional is

$$
Z_{C T P}\left[J_{+}, J_{-}\right]=\exp \left(-\frac{i}{4} J_{+}^{x} \cdot \dot{j}_{+}^{p}+\frac{i}{4} J_{-}^{x} \cdot \dot{J}_{-}^{p}\right),
$$

where $J_{ \pm}^{x}$ and $J_{ \pm}^{p}$ are the source terms for $\dot{x}$ and $\dot{p}$ respectively.

It is interesting to write the quantum differential equation for a particle with a Hamiltonian $\hat{H}=\frac{\hat{p}^{2}}{2 m}+V(\hat{x})$. If we denote as $\xi_{t}^{x}=\dot{X}_{t}$ and $\xi_{t}^{p}=\dot{P}_{t}$ the external sources associated with the kinematic process, the quantum differential equations read

$$
\begin{aligned}
\dot{x}-\frac{1}{m} p & =\xi_{t}^{x} \\
\dot{p}+V^{\prime}(x) & =\xi_{t}^{p},
\end{aligned}
$$

which implies that

$$
\ddot{x}+V^{\prime}(x)=\Xi_{t},
$$

where

$$
\Xi_{t}=\dot{\xi}_{t}^{x}-\frac{1}{m} \xi_{t}^{p}
$$

This implies that the external force for the configuration space equation of motion is a measure of the failure of the momentum to coincide with particle velocity in the kinematic process, something anticipated in [23]. It is interesting to also remark that if we consider the case of fields, we can still write the analogue of the quantum differential equation on configuration space. For a scalar field this would be

$$
\ddot{\phi}-\nabla^{2} \phi-m^{2} \phi-V^{\prime}(\phi)=\Xi_{t}
$$

While the left-hand side is the deterministic Lorentz invariant equation of motion, the right-hand side, which contains the quantum mechanical contribution, explicitly depends on the choice of time variable through the choice of momentum and hence the quantum differential equation does depend on the choice of the spacetime foliation. This fact is not apparent (but still present) in standard quantum theory, has been identified by Savvidou in the context of continuoustime histories [18].

Before concluding this section, we want to remark on the appealing possibility that equation (4.43) can be interpreted as referring to an individual system in analogy to the classical Langevin equations. That is, we can consider that equation (4. 44) refers to an individual system (a particle), which is found within a "fluctuating environment", that induces the "random forces" 
$\xi^{a}(t)=\dot{F}_{t}^{a}$. However, these forces are not distributed according to a classical probability distribution, but according to the kinematic processes (and are possibly geometrical in origin).

We are not in a position to argue, whether this interpretation should be taken seriously or not. The reasons are partly mathematical and partly physical: from the mathematical side we need to verify that equations such as (4. 44) are more than empty symbols: is it actually a type of equation that can admit solutions? we hope to justify such equations by adopting the theory of stochastic integrals (of Ito) in the quantum context. From a physical point of view, even though we are committed to finding a description for the individual quantum system, the picture of a particle moving under random forces is not necessarily our first choice: it is perhaps too classical. Nonetheless, equation (4. 44) has large theoretical interest and we would like to see, whether it would be possible to simulate its solutions numerically as we can do with stochastic processes. This would provide a way of generating actual trajectories for individual quantum systems.

\section{$5 \quad$ Recovering quantum mechanics}

In the previous section, we showed how to obtain quantum processes starting from quantum theory. Now, we want to invert this procedure and ask how one can obtain standard quantum theory starting from a generic quantum process, that satisfies the axioms stated in section 4.1.

\subsection{The Markov property for quantum processes}

One important feature of quantum dynamics is that the time evolution of the object encoding the probabilities (the wave function or the density matrix) is given by a linear partial differential equation; this means that the knowledge of this object at a moment of time suffices to determine it at any future instant.

The analogous property for classical processes is known as the Markov property. We shall here try to study in more detail its quantum analogue.

\subsubsection{Conditioning with respect to past}

Let us consider a quantum process $\left(\Omega, \Phi, F_{t}^{a}\right)$, where we choose the $F_{t}^{a}$ to correspond to a complete set of coordinate functions on the single-time manifold $\Gamma$. We will denote $F_{t}: \Omega \rightarrow \mathbf{R}^{n}$.

We can define two subfields. One is the instant subfield $\mathcal{A}_{t}$, which is generated by all sets of the form $\left(F_{t}\right)^{-1}(B)$, for a fixed value of $t$ and any Borel subset $B$ of $\mathbf{R}^{n}$. The other is the past subfield $\mathcal{A}_{\leq t}$, which is generated by all sets of the form $\left(F_{s}\right)^{-1}(B)$. for $t_{0} \leq s \leq t$ and Borel subsets $B$ of $\mathbf{R}^{n}$. 
The Markov property is phrased in terms of the conditional pairs. If we have the times $t, t^{\prime} \geq s \geq t_{0}$, then a process $\left(\Omega, \Phi, F_{t}^{a}\right)$ satisfies the Markov property if

$$
\tilde{\Phi}\left(\left(F_{t}\right)^{-1}(C),\left(F_{t^{\prime}}\right)^{-1}\left(C^{\prime}\right) \mid \mathcal{A}_{s}\right)=\tilde{\Phi}\left(\left(F_{t}\right)^{-1}(C),\left(F_{t^{\prime}}\right)^{-1}\left(C^{\prime}\right) \mid \mathcal{A}_{\leq s}\right),
$$

for any Borel sets $C$ and $C^{\prime}$ in $\mathbf{R}^{n}$.

This states that once we have the full knowledge of the decoherence functional at a moment $t$, we need no knowledge from its past in order to define probabilities and phases for any future measurements.

\subsubsection{The quantum Chapman-Kolmogorov equation}

Now $\tilde{\Phi}\left(\left(F_{t}\right)^{-1}(C),\left(F_{t^{\prime}}\right)^{-1}\left(C^{\prime}\right) \mid \mathcal{A}_{s}\right)$ is a functional on $\mathbf{R}^{n} \times \mathbf{R}^{m}$, and can be written in terms of the Lebesque measure $d^{n} z_{t}$ in $\mathbf{R}^{m}$, which induced through the coordinate functions $F_{t}^{a}$ from the measure $d z$ on $\Omega$. Explicitly we would have

$$
\tilde{\Phi}\left(\left(F_{t}\right)^{-1}(C),\left(F_{t^{\prime}}\right)^{-1}\left(C^{\prime}\right) \mid \mathcal{A}_{s}\right)=\int \chi_{C}(z) \chi_{C^{\prime}}\left(z^{\prime}\right) v\left(z, t ; z^{\prime}, t^{\prime} \mid \mathcal{A}_{s}\right) d^{n} z_{t} d^{n} z_{t^{\prime}}
$$

Now, we have

$$
\begin{array}{r}
\tilde{\Phi}\left(\left(F_{t}\right)^{-1}(C),\left(F_{t^{\prime}}\right)^{-1}\left(C^{\prime}\right) \mid \mathcal{A}_{s}\right)=\tilde{\Phi}\left(\chi_{C}\left(F_{t}\right), \chi_{C^{\prime}}\left(F_{t^{\prime}}\right) \mid \mathcal{A}_{s}\right) \\
=\tilde{\Phi}\left(\chi_{C}\left(F_{t}\right), \chi_{C^{\prime}}\left(F_{t^{\prime}}\right) \mid \mathcal{A}_{\leq s}\right)=\tilde{\Phi}\left[\tilde{\Phi}\left(\chi_{C}\left(F_{t}\right), \chi_{C^{\prime}}\left(F_{t^{\prime}}\right) \mid \mathcal{A}_{\leq s}\right) \mid \mathcal{A}_{\leq s^{\prime}}\right],
\end{array}
$$

where $s^{\prime} \geq s$, by virtue of (3. 24) and since obviously $\mathcal{A}_{\leq s} \subseteq \mathcal{A}_{\leq s^{\prime}}$ if $s \leq s^{\prime}$. (Note the use of the Markov property in equation (5. 3).)

We can further work on this equation to get

$$
\begin{array}{r}
\tilde{\Phi}\left(\left(F_{t}\right)^{-1}(C),\left(F_{t^{\prime}}\right)^{-1}\left(C^{\prime}\right) \mid \mathcal{A}_{s}\right)=\tilde{\Phi}\left[\tilde{\Phi}\left(\chi_{C}\left(F_{t}\right), \chi_{C^{\prime}}\left(F_{t^{\prime}}\right) \mid \mathcal{A}_{\leq s^{\prime}}\right) \mid \mathcal{A}_{\leq s}\right] \\
=\tilde{\Phi}\left[\tilde{\Phi}\left(\chi_{C}\left(F_{t}\right), \chi_{C^{\prime}}\left(F_{t^{\prime}}\right) \mid \mathcal{A}_{s^{\prime}}\right) \mid \mathcal{A}_{s}\right]
\end{array}
$$

Now let us recall that $\tilde{\Phi}\left(\cdot, \cdot \mid \mathcal{A}_{s}\right)=\tilde{\Phi}\left(\cdot, \cdot \mid F_{s}\right)$ is a function on $\Omega \times \Omega$. Since $F_{s}$ are coordinate functions and locally in one-to-one correspondence with points of $\Gamma_{s}$ one can write this as

$$
\tilde{\Phi}\left(\cdot, \cdot \mid z, z^{\prime}, s\right)
$$

Hence, the corresponding densities $v\left(z, t ; z^{\prime}, t^{\prime} \mid \mathcal{A}_{s}\right)$ can be represented by a kernel with arguments on $\Gamma$ as $v\left(z_{1}, t ; z_{1}^{\prime}, t^{\prime} \mid z_{0}, z_{0}^{\prime}, s\right)$. Taking $t=t^{\prime}$ we can define the function

$$
v\left(z_{1}, z_{1}^{\prime} ; t \mid z_{0}, z_{0}^{\prime} ; s\right)
$$

which is essentially the density matrix propagator.

If we write $\tilde{\Phi}$ in terms of this kernel in equation (5.4) we will obtain

$$
v\left(z_{1}, z_{1}^{\prime} ; t \mid z_{0}, z_{0}^{\prime} ; s\right)=\int d z d z^{\prime} v\left(z_{1}, z_{1}^{\prime} ; t \mid z, z^{\prime} ; s^{\prime}\right) v\left(z, z^{\prime} ; s^{\prime} \mid z_{0}, z_{0}^{\prime} ; s\right)
$$


This property of Markov processes is a quantum version of the ChapmanKolmogorov equation.

The quantum Chapman-Kolmogorov equations implies, that the knowledge of the propagator $v$ together with the decoherence functional at the initial moment of time suffices to determine the full hierarchy of distribution functions.

Indeed, if we get a density $\rho_{0}\left(z_{0}, z_{0}^{\prime}\right)$ for time $t=0$, one can determine the diagonal distribution functions

$$
\begin{array}{r}
v^{N+1, N+1}\left(z_{0}, t_{0} ; z_{1}, t_{1} ; \ldots ; z_{N}, t_{N-1} \mid z_{0}^{\prime}, t_{0} ; z_{1}, t_{1} ; \ldots ; z_{N-1}^{\prime}, t_{N-1}\right)= \\
v\left(z_{N}, z_{N}^{\prime} ; t_{N} \mid z_{N-1}, z_{N-1}^{\prime} ; t_{N-1}\right) \ldots v\left(z_{1}, z_{1}^{\prime} ; t_{1} \mid z_{0}, z_{0}^{\prime} ; t_{0}\right) \rho_{0}\left(z_{0}, z_{0}^{\prime}\right) .
\end{array}
$$

As we explained in section 4.1 these distribution functions contain enough information to determine the hierarchy $v^{n, m}$ and hence the decoherence functional.

\subsubsection{Symmetries of the propagator}

By virtue of its definition and the properties of the decoherence functional it is easy to demonstrate that the propagator satisfies the following properties

1. Hermiticity: $v\left(z, z^{\prime} ; t \mid z_{0}, z_{0}^{\prime} ; s\right)=\bar{v}\left(z^{\prime}, z ; t \mid z_{0}^{\prime}, z_{0} ; s\right)$.

This is clearly a consequence of the hermiticity property of the decoherence functional and is inherited into the conditional pair, by which the propagator is defined.

2. Normalisation: $\int d z d z^{\prime} v\left(z, z^{\prime} ; t \mid z_{0}, z_{0}^{\prime} ; s\right)=1$.

This follows from the normalisation condition, by virtue of (3. 25) .

3. Positivity: If $\rho_{0}$ is positive, then so is $\int d z_{0} d z_{0}^{\prime} v\left(z, z^{\prime} ; t \mid z_{0}, z_{0}^{\prime} ; s\right) \rho_{0}\left(z_{0}, z_{0}^{\prime}\right)$. A function $\rho\left(z, z^{\prime}\right)$ is called positive, if it satisfies the positivity condition of the decoherence functional, i.e. for any (complex-valued) function $F$,

$$
\int d z d z^{\prime} \rho\left(z, z^{\prime}\right) F(z) F^{*}\left(z^{\prime}\right) \geq 0 .
$$

It is clear that this condition follows from the positivity property of the decoherence functional.

These properties of the decoherence functional can be written in an operator language. For this purpose, let us consider the Hilbert space $V=\mathcal{L}^{2}(\Gamma, d z)$ and its dual $\bar{V}$. This is not the physical Hilbert space of the corresponding quantum theory; it is simply introduced as a convenient way to describe the conditions 1-3 for the propagator.

We shall denote elements of $V$ as $f_{\rightarrow}$, of $\bar{V}$ as $g_{\leftarrow}$ and as $g_{\leftarrow} \cdot f_{\rightarrow}$ the operation of the natural pairing between $V$ and $\bar{V}$. We employ the arrows in order to keep track in which Hilbert space each element belongs. 
In $V \otimes \bar{V}$ we have the vectors $\mid f g)=f_{\rightarrow} \otimes g_{\leftarrow}$, which have as inner product

$$
\left(f^{\prime} g^{\prime} \mid f g\right)=\left(f_{\rightarrow}^{\prime} \cdot f_{\leftarrow}\right)\left(g_{\rightarrow} \cdot g_{\leftarrow}^{\prime}\right)
$$

For fixed values of $t$ and $s, v$ defines an operator $Y$ on $V \otimes \bar{V}$, such that

$$
\left(f^{\prime} g^{\prime}|Y| f g\right)=\int d z d z^{\prime} d z_{0} d z_{0}^{\prime} \bar{f}^{\prime}(z) g^{\prime}\left(z^{\prime}\right) v\left(z, z^{\prime} ; t \mid z_{0}, z_{0}^{\prime} ; s\right) f\left(z_{0}\right) \bar{g}\left(z_{0}^{\prime}\right),
$$

for functions $f, g, f^{\prime}, g^{\prime}$ that are elements of $V$.

Now the pairing · can be extended by linearity to a linear map from $V \otimes \bar{V}$ to $\mathbf{C}$. Given the fact that $V \otimes \bar{V}$ is a subspace of the space of bounded linear operators $B(V)$ on $V$, the pairing - is identical to the trace functional on $B(V)$. The normalisation condition implies that $Y$ preserves the map ·, or in other words if $Y$ is viewed as an operator on $B(V)$ is trace-preserving.

Now $V \otimes \bar{V}$ is the space of Hilbert-Schmidt operators on $H^{4}$ and if $O$ is such an operator $Y$ will act in such a way as

$$
\operatorname{Tr} Y(O)=\operatorname{Tr} O .
$$

This relation is generalised to further operators besides the Hilbert-Schmidt ones by linearity and taking limits with respect to the trace-norm topology.

The hermiticity condition, when applied to such an operator $Y$ implies that

$$
\operatorname{Tr}\left(A^{\dagger} Y(B)\right)=\operatorname{Tr}\left[A Y\left(B^{\dagger}\right)\right]
$$

for Hilbert-Schmidt operators $A$ and $B$.

The positivity condition is translated to the fact, that if $O$ is a positive operator then $Y(O)$ is also positive.

One way to see the physical meaning of these conditions is to consider the class of trace-preserving operators of the form $Y(O)=\sum_{i} c_{i} A_{i} O A_{i}^{-1}$, for some operators $A_{i}$ acting on $V$ and complex coefficients $c_{i}$ such that $\sum_{i} c_{i}=1$. (Almost all trace-preserving operators are of this form). The hermiticity condition implies that $A_{i}$ are unitary, while the positivity condition that $c_{i}$ are non-negative. So $Y$ is a convex combination of unitary automorphisms

$$
Y(O)=\sum_{i} c_{i} U_{i} O U_{i}^{\dagger}
$$

for a family of unitary operators $U_{i}$.

\subsubsection{Time symmetries}

Now, there are certain properties concerning the temporal properties of the quantum process that can be written as symmetries of the propagator $v$.

\footnotetext{
${ }^{4} \mathrm{~A}$ Hilbert-Schmidt operator is one that has finite value of the norm $\|A\|_{2}=\left[\operatorname{Tr}\left(A^{\dagger} A\right)\right]^{1 / 2}$.
} 
Time homogeneity: A quantum Markov process is called time homogeneous if $v\left(z, z^{\prime} ; t \mid z_{0}, z_{0}^{\prime} ; s\right)=v\left(z, z^{\prime} ; t \mid z_{0}, z_{0}^{\prime} ; 0\right)$. This implies that the information of the propagator is contained in the one-parameter family of kernels $v_{t}\left(z, z^{\prime} \mid z_{0}, z_{0}^{\prime}\right)$. Note, that in the physics literature, it is often the time-homogeneous Markov processes that are referred to as Markov processes.

If we translate this condition in terms of the operator $Y$ on $V \otimes \bar{V}$, it implies that $Y_{t}$ forms an one-parameter group of trace-preserving transformations.

More important is the following condition

Time reversibility: A quantum Markov process in an interval $\left[t_{0}, t_{f}\right]$ is called time-reversible if

$$
v\left(z, z^{\prime} ;\left(t_{f}-t_{0}\right)-t \mid z_{0}, z_{0}^{\prime} ;\left(t_{f}-t_{0}\right)-s\right)=\bar{v}\left(z, z^{\prime} ; t \mid z_{0}, z_{0}^{\prime} ; s\right)
$$

This condition implies that one can interpret the two entries of the decoherence functional as a path going forward in time and one going backwards, implying that its evaluation takes place in a closed time-path.

For this purpose one could write more naturally the condition of strong time reversibility

$$
\begin{array}{r}
v_{n, m}\left(z_{1}, t_{1} ; \ldots ; z_{n}, t_{n} \mid z_{1}^{\prime}, t_{1}^{\prime} ; \ldots ; z_{m}^{\prime}, t_{m}^{\prime}\right)= \\
\bar{v}^{n, m}\left(z_{1}^{\prime}, t_{1}^{\prime} ; \ldots ; z_{m}^{\prime}, t_{m}^{\prime} \mid z_{1}, t_{1} ; \ldots ; z_{n}, t_{n}\right)
\end{array}
$$

This is, however, too strong: all physical decoherence functionals break time reversibility by virtue of containing information about an initial condition at a single moment of time. The only way to satisfy this is with a decoherence functional containing both initial and final conditions, and such that these conditions are in some sense equivalent ${ }^{5}$. For this reason, the condition (5. 15) which singles out the dynamical notion of time-reversibility is preferred. We should note, a separation between dynamics and initial condition makes sense only when the Markov property is satisfied.

The condition of time-reversibility implies that the operator $Y$ on $V \otimes \hat{V}$ is unitary, i.e. that

$$
\operatorname{Tr}\left(A^{\dagger} Y(B)\right)=\operatorname{Tr}\left(Y^{-1}(A)^{\dagger} B\right)
$$

Now, if $A$ and $B$ are the one-dimensional projector $P$ this condition implies that

$$
\operatorname{Tr}\left(Y(P)^{\dagger} Y(P)\right)=\operatorname{Tr}\left(P^{2}\right)=\operatorname{Tr} P=1 .
$$

\footnotetext{
${ }^{5}$ In Hilbert space quantum theory one can insert a final-time density matrix $\hat{\rho}_{f}$ in equation
} (2. 2). The theory is then strongly time reversible if $\hat{\rho}_{f}=e^{-i \hat{H}\left(t_{f}-t_{0}\right)} \hat{\rho}_{0} e^{i \hat{H}\left(t_{f}-t_{0}\right)}$. Such theories are not employed as all preparations of the system (by which we condition and define the initial state) take place in the past of the measuring procedure. This construction is not nonsensical, but (at least seemingly) void of any operational content. If, however, we decide to extend the scope of quantum theory at the realm of cosmology, such conditions cannot be excluded and they may hold an appeal because of their strong symmetry. 
This implies that $Y$ preserves pure density matrix and it is a theorem [39] that it should be of the form

$$
Y(O)=U O U^{\dagger}
$$

for some unitary operator $U$. One way to see this without referring to the lengthy proof is to notice that operators of the form (5. 14) satisfy property (5. $17)$ if and only if $c_{i}=0$ for all but one values of $i$. This does not constitute a proof, however, because not all operators that satisfy the hermiticity, tracepreservation and positivity properties can be written in the form (5. 14).

In any case, time reversibility implies that $Y=U \otimes U^{\dagger}$. This means that the propagator $v$ factorises

$$
v\left(z, z^{\prime} ; t \mid z_{0}, z_{0}^{\prime} ; s\right)=\chi\left(z, t \mid z_{0}, s\right) \bar{\chi}\left(z^{\prime}, t^{\prime} \mid z_{0}^{\prime}, s\right)
$$

for a kernel $\chi$ on $\Gamma_{t} \times \Gamma_{s}$, that corresponds to the unitary operator $Y$. This clearly satisfies

$$
\chi\left(z, t \mid z_{0}, s\right)=\bar{\chi}\left(z_{0}, s \mid z, t\right) .
$$

This kernel will be eventually interpreted as the wave function propagator in the coherent state basis. If, in addition, the process is time homogeneous one can write

$$
\chi\left(z, t \mid z_{0}, s\right)=\chi_{t-s}\left(z \mid z_{0}\right),
$$

in terms of an one-parameter family of kernels $\chi_{t}$ on $\Gamma \times \Gamma$.

In a nutshell, a quantum process that satisfies the Markov property is completely characterised by the knowledge of a single time kernel $v_{0}$ and of the propagator $v$. If it is time-symmetric the propagator factorises and is determined in terms of a single kernel $\chi$ on $\Gamma_{t} \times \Gamma_{s}$.

\subsection{Deriving quantum theory}

We will now show how the Hilbert space of standard quantum theory naturally arises in the study of quantum processes. First let us emphasise once more, that the Hilbert space $V$ we employed in the previous section is not the Hilbert space of standard quantum theory. The latter is actually a subspace of $V$ and it is, in general, non-trivial to determine how this subspace would be selected.

\subsubsection{The physical Hilbert space and the Hamiltonian}

Now, let us suppose that we have a quantum process $\left(\Omega, \Phi, F_{t}^{a}\right)$, where $\Omega$ is a subset of $x_{t} \Gamma_{t}, \Gamma$ is a manifold (to be identified with the classical phase space) and $F_{t}^{a}$ correspond to coordinates on $\Gamma$. Assume further that this process satisfies the Markov property.

As we showed previously the Markov property implies that the process is uniquely determined by the knowledge of a kernel $v_{0}\left(z_{0}, z_{0}^{\prime}\right)$ at some reference time $t_{0}$ and the propagating kernel $v\left(z, z^{\prime} ; t \mid z_{0}, z_{0}^{\prime} ; s\right)$. If we also assume that 
the process is time reversible, then one needs simply to specify a propagating kernel of the type $\chi\left(z, t \mid z_{0}, s\right)$.

Let us now study the kinematical process associated with this quantum process by considering the decoherence functional generated by $v_{0}$ and a propagating kernel $\psi_{t}\left(z \mid z^{\prime}\right)=\lim _{s \rightarrow t} \chi\left(z, t \mid z^{\prime}, s\right)$. We need to assume that $\psi_{t}$ is a continuous and perhaps sufficiently smooth function on $\Gamma^{6}$.

The quantum Chapman-Kolmogorov equation is valid for the kinematical process also so

$$
\psi_{t}\left(z \mid z^{\prime}\right)=\int d z^{\prime \prime} \psi_{t}\left(z \mid z^{\prime \prime}\right) \psi_{t}\left(z^{\prime \prime}, z^{\prime}\right)
$$

This is, however, the defining equation for a projection operator $E_{t}$ in the space $V=\mathcal{L}^{2}(\Omega, d z)$. The range of this projector is a Hilbert space $H_{t}$, corresponding to the instant $t$.

If the kinematical process is time-homogeneous, i.e. $\psi_{t}$ does not depend on the time $t$, then all $H_{t}$ are naturally identified with a reference Hilbert space $H$, which is the Hilbert space of standard quantum theory.

Let us now consider the kernel $\chi_{t}\left(z \mid z^{\prime}\right)$. The quantum Chapman-Kolmogorov equation implies that

$$
\chi_{t}\left(z \mid z^{\prime}\right)=\int d z^{\prime \prime} \psi\left(z \mid z^{\prime \prime}\right) \chi_{t}\left(z^{\prime \prime} \mid z^{\prime}\right)=\int d z^{\prime \prime} \chi_{t}\left(z \mid z^{\prime \prime}\right) \psi\left(z^{\prime \prime} \mid z^{\prime}\right)
$$

In terms of operators on $V$, this reads

$$
U_{t}=E U_{t}=U_{t} E,
$$

implying that $U_{t}=E U_{t} E$. The kernel $\chi_{t}$ then defines an one-parameter family of unitary operators on $H$; having assumed continuity at $t=0$, we can employ Stone's theorem to determine that it would be of the form $e^{-i \hat{H} t}$ in terms of a self-adjoint operator on $H$.

We need to repeat again that the finiteness and continuity of $\chi_{t}$ is necessary if we are to obtain the Hilbert space $H$ and the corresponding Hamiltonian. In absence of this condition, there is no way to pass from the unphysical Hilbert space $V$, to the Hilbert space $H$.

Some further remarks can be made at this point. Consider the kernel $\rho_{0}$ containing the information about the initial state. It corresponds to an operator $\hat{\rho}_{0}$ on $V$. Since the restriction of the decoherence functional at $t_{0}$ still has to satisfy the axioms of the decoherence functional, we see that $\hat{\rho}_{0}$ is a density matrix on $V$. Now, any integration over $z_{i}$ or $z_{i}^{\prime}$ in equation (5. 8) (for the determination of a correlation function) will essentially amount to squeezing $\hat{\rho}_{0}$ between two projectors $E$ as $E \hat{\rho}_{0} E$. This means that effectively the initial condition will be encoded in a density matrix on $H$, rather than $V$, which is the standard result.

\footnotetext{
${ }^{6}$ Note if we study the standard quantum process on configuration space the kernel $\psi_{t}$ defining the kinematical process is a $\delta$-function, which cannot straightforwardly define a quantum process. This is unlike the case of phase space processes we consider here.
} 


\subsubsection{The coherent state picture}

Now, on $V$ one can write the coherent states $\Psi_{z}$ as the functions $\Psi_{z}\left(z^{\prime}\right)=$ $\chi\left(z^{\prime} \mid z\right)$. The whole Hilbert space $H$ can be obtained from finite linear combinations of these coherent states. Clearly then, $\chi_{t}\left(z, z^{\prime}\right)=\left\langle z^{\prime}\left|e^{-i \hat{H} t}\right| z\right\rangle$ and the distribution functions $v^{n, m}$ will read

$$
\begin{array}{r}
v_{z_{0}}^{n, m}\left(z_{1}, t_{1} ; z_{2}, t_{2} ; \ldots ; z_{n}, t_{n} \mid z_{1}^{\prime}, t_{1}^{\prime} ; z_{2}^{\prime}, t_{2}^{\prime} ; \ldots ; z_{m}^{\prime}, t_{m}^{\prime}\right)= \\
\left\langle z_{m}^{\prime}\left|e^{-i \hat{H}\left(t_{m}^{\prime}-t_{n}\right)}\right| z_{n}\right\rangle\left\langle z_{n}\left|e^{-i \hat{H}\left(t_{n}-t_{n-1}\right)}\right| z_{n-1}\right\rangle \ldots \\
\times\left\langle z_{2}\left|e^{-i \hat{H}\left(t_{2}-t_{1}\right)}\right| z_{1}\right\rangle\left\langle z_{1}\left|e^{-i \hat{H}\left(t_{1}-t_{0}\right)}\right| z_{0}\right\rangle\left\langle z_{0}\left|\hat{\rho}_{0}\right| z_{0}^{\prime}\right\rangle \\
\times\left\langle z_{0}^{\prime}\left|e^{i \hat{H}\left(t_{1}^{\prime}-t_{0}\right)}\right| z_{1}^{\prime}\right\rangle\left\langle z_{1}^{\prime}\left|e^{i \hat{H}\left(t_{2}^{\prime}-t_{1}^{\prime}\right)}\right| z_{2}^{\prime}\right\rangle \ldots\left\langle z_{n-1}^{\prime}\left|e^{i \hat{H}\left(t_{n}^{\prime}-t_{n-1}^{\prime}\right)}\right| z_{n}^{\prime}\right\rangle,
\end{array}
$$

which is the standard quantum mechanical result.

Furthermore if we consider functions $f^{a}$ on $\Gamma$, we can write the correlation functions for the process corresponding to $F_{t}^{a}$

$$
\begin{gathered}
G^{n, m}\left(a_{1}, t_{1} ; \ldots ; a_{n}, t_{n} \mid a_{1}^{\prime}, t_{1}^{\prime} ; \ldots ; a_{m}^{\prime}, t_{m}^{\prime}\right)= \\
\operatorname{Tr}\left(\hat{A}^{a_{n}}\left(t_{n}\right) \ldots \hat{A}^{a_{1}}\left(t_{1}\right) \hat{\rho}_{0} \hat{A}^{a_{1}^{\prime}}\left(t_{1}^{\prime}\right) \ldots \hat{A}^{a_{m}^{\prime}}\left(t_{m}^{\prime}\right)\right) .
\end{gathered}
$$

In this equation we have assume temporal ordering as $t_{1}<t_{2}<\ldots<t_{n}$ and $t_{1}^{\prime}<t_{2}^{\prime}<\ldots<t_{m}^{\prime}$. The operators $\hat{A}(t)=e^{i \hat{H}\left(t-t_{0}\right)} \hat{A} e^{-i \hat{H}\left(t-t_{0}\right)}$ are the Heisenberg picture operators and

$$
\hat{A}^{a}=\int d z f^{a}(z)|z\rangle\langle z|
$$

Furthermore, if we have a pair of discrete histories $\alpha=\left(C_{1}, t_{1} ; \ldots ; C_{n}, t_{n}\right)$ and $\beta=\left(C_{1}^{\prime}, t_{1}^{\prime} ; \ldots ; C_{m}^{\prime}, t_{m}^{\prime}\right)$, where the $C_{i}$ and $C_{i}^{\prime}$ are measurable subsets of $\Gamma$, we will have the value of the decoherence functional

$$
\Phi(\alpha, \beta)=\operatorname{Tr}\left(\hat{C}_{n}\left(t_{n}\right) \ldots \hat{C}_{1}\left(t_{1}\right) \hat{\rho}_{0} \hat{C}_{1}^{\prime}\left(t_{1}^{\prime}\right) \ldots \hat{C}_{m}^{\prime}\left(t_{m}^{\prime}\right)\right),
$$

where here $\hat{C}=\int_{C} d z|z\rangle\langle z|$ are positive operators that represent the phase space cell $C$. They have been used in [40] in the discussion of the classical limit. Again $\hat{C}(t)$ denotes the Heisenberg-picture operator.

This expression for the decoherence functional is identical with the standard expression (2. 2); the only difference is that in our case events are not represented by projectors. Events correspond to measurable subsets of $\Gamma$, which form a Boolean algebra and as such they are represented by a particular class of positive operators.

The assumption of time-reversibility is not essential in obtaining standard results of quantum theory. One can construct a kinematical process corresponding to $v\left(z, z^{\prime} ; t \mid z_{0}, z_{0}^{\prime} ; s\right)$. In the case of a time-homogeneous process the corresponding kernel defines a projection operator into a closed linear subspace $U$ of $V \otimes \bar{V}$, 
which satisfies the hermiticity property. Similarly the dynamics correspond to an operator that is projected on $U$; effectively $Y_{t}$ corresponds to a bistochastic map characterising an open quantum system [22].

\subsubsection{A summary}

Let us recapitulate the results, so far, in this section. If we assume we have a quantum process that

1. Satisfies the Markov property,

2. Its propagator $v$ is a continuous function of the time variable as well as the points $z \in \Gamma$,

then we recover standard quantum theory for open systems (of the Markov type).

3. If in addition we demand that the quantum process is time-reversible

we can define a Hilbert space for each moment of time, but it is not necessary that this can be reduced to the standard case of a single Hilbert space describing the whole system. We have analysed this feature of quantum mechanical histories (with reference to quantum field theory in curved spacetime) in [41].

4. If we also demand that the quantum process is time-homogeneous,

we can write a reference Hilbert space describing the system at all times, verify that the dynamics are given by a Hamiltonian operator and obtain standard quantum theory with all its predictions but one.

Namely we get the correct correlation functions for the observables of quantum theory, but our events do not correspond to projection operators. They correspond to a larger class of positive, bounded operators ${ }^{7}$.

In other words the theory of quantum processes gives an equivalent description of the one afforded to quantum theory (as far as statistical predictions are concerned), while it does not abandon the classical "logic" for the description of events.

\subsubsection{Some remarks}

At this point we need to make a number of important remarks

1. The fact that the physical Hilbert space is not identical with the mathematically natural one $V$, is due to the fact that $\psi\left(z \mid z^{\prime}\right)$ is a genuine function

\footnotetext{
${ }^{7}$ One can easily check that in terms of the supremum norm $\|\hat{C}\| \leq 1$.
} 
over $\Gamma \times \Gamma$. Thus it can be used to define a projector. If this were not the case and $\psi$ were a delta function the corresponding projector would be unity, and $V$ would be identical with $H$. Such is the case in classical probability theory; in this case the kinematic process is completely trivial. Hence one might say that the non-triviality of the kinematical process is the reason, why the physical Hilbert space is a subspace of $V$.

2. In equation (4. 22) we chose to employ the $P$-symbol as giving the correspondence between quantum mechanical operators and phase space functions. This choice was at that point arbitrary; there is a continuous infinity of possible symbols one could have employed for the correspondence, the $P$-symbols being just one possibility. However, in our analysis of section 5 , the $P$-symbol arises naturally as giving the correspondence between functions and operators. In our argumentation, we started with a quantum process on phase space, hence the functions $f^{a}(z)$ are the fundamental variables and the operators are secondary objects; given the way operators appear as a convenient description, equation (4. 22) for the $P$-symbol is the only one that physically makes sense.

3. In defining the quantum Markov property we have imposed a condition on conditional pairs, which is the mathematically natural thing to do. However, the incorporation of measurement outcomes in the decoherence functional takes place with respect to conditional correlation, as this is equivalent to the "reduction of the wave packet", and this object is too unwieldy to yield a useful definition of a Markov process. This distinction is in contrast to classical probability theory, where the conditional expectation is used for both defining the Markov property and incorporating experimental results. This difference points to a fundamental difference of the notion of events between classical and quantum theory (whenever we attempt to have a realist description of the theory). This is not an issue that we can further elaborate at this point.

4. Standard quantum mechanics is recovered only when we have a Markov process. This is natural as the notion of state makes little sense otherwise; $a$ state is a description of the system at a moment of time. It contains information about possible measurements at this instant, But it should also contain information that will allow us to determine (or predict) the state at subsequent moments of time (assuming we know the dynamical law). In absence of the Markov property, no information at a moment of time suffices to determine the future development of the system.

\subsection{Phase space structures in quantum processes}

We have seen that a time-reversible, Markov quantum process can be determined by the propagator $\chi_{t}\left(z \mid z^{\prime}\right), z, z^{\prime} \in \Gamma$. The question then arises, how such an object can be constructed solely from geometric structures on the symplectic 
manifold $\Gamma$. One answer comes from the coherent state quantisation programme developed by Klauder [42].

The idea is that the propagator in the coherent state basis can be obtained as a suitably regularised path integral. The regularisation takes place with respect to a homogeneous' Riemannian metric $g$ on $\Gamma$. This metric can be used to define a conditioned Wiener process on $\Gamma$, with fixed points at initial and final moments $z(0)=z_{0}$ and $z(t)=z_{f}$. The corresponding reads formally

$$
d \mu_{z_{0}, z_{f}}^{\nu}[z(\cdot)]=\exp \left(-\frac{1}{2 \nu} \int_{0}^{t} g(\dot{z}, \dot{z}) d s\right) D z(\cdot) .
$$

Then it turns out that one can write

$$
\chi_{t}\left(z_{f} \mid z_{0}\right)=\lim _{\nu \rightarrow \infty} 2 \pi e^{\nu t / 2} \int e^{i\left(\int_{z(\cdot)} A-\int_{0}^{t} h(z(s)) d s\right)} d \mu_{z_{0}, z_{f}}^{\nu}[z(\cdot)] .
$$

In this expression $A$ is a connection one-form such that $d A=\omega$, with $\omega$ the symplectic form on $\Gamma$. Such an one-form arises from the procedure of geometric quantisation, i.e. identifying a $U(1)$ bundle over $\Gamma$, with a connection whose curvature projects to $\omega$. The Hamiltonian $h(z)$ is essentially the $P$-symbol of the quantum Hamiltonian operator.

As Klauder has emphasised the whole procedure is fully geometrical; if we want to construct the projection operator $E$ that defines the physical Hilbert space we need to specify the connection $A$ and the Riemannian metric, since

$$
\psi\left(z_{f} \mid z_{0}\right)=\lim _{\nu \rightarrow \infty} 2 \pi e^{\nu t / 2} \int e^{i \int_{z(\cdot)} A} d \mu_{z_{0}, z_{f}}^{\nu}[z(\cdot)] .
$$

In order to specify the dynamical component we need to specify a scalar function on $\Gamma$ that will act as the Hamiltonian.

\section{Interpretational issues}

As we explained, the difference between quantum processes and standard quantum theory lies only in the determination of which object correspond to sharp events. Quantum mechanics admits projection operators, while the theory of quantum processes admits phase space cells. These are represented by a positive operator-valued-measure $C \rightarrow \hat{C}=\int_{C} d z|z\rangle\langle z|$, for any measurable subset $C$ of $\Gamma$.

The question then arises, which of the basic principles of quantum theory is (are ) violated by this change and whether this violation has empirical consequences. 


\subsection{Comparing with standard quantum theory}

Many textbooks employ the following basic principles (or some variations of them) as axioms, out of which the basic structure of quantum theory is derived.

D1. States: A state of the system at a moment of time is represented by a vector on a Hilbert space $H$, or a density matrix on $H$.

D2. Observables: Observables are represented by self-adjoint operators on $H$.

D3. Properties: The possible values for an observable correspond to the points of the spectrum of the corresponding operator. As a corollary a proposition about possible values of an observable is represented by a projection operator. D4. Probabilities: If $\hat{P}$ is a projection operator representing a property, then the probability that this is true in a state $\hat{\rho}$ equals $\operatorname{Tr}(\hat{\rho} \hat{P})$.

D5. Combination of subsystems: If $H_{1}$ and $H_{2}$ are Hilbert spaces characterising independent systems, the combined system is characterised by $H_{1} \otimes H_{2}$.

D6. Conditioning: If an experiment verifies a property corresponding to a projection operator $\hat{P}$, then we encode this information by transforming the state as $\hat{\rho} \rightarrow \hat{P} \hat{\rho} \hat{P} / \operatorname{Tr}(\hat{\rho} \hat{P})$.

D7. Time evolution: If the system is left isolated, its state evolves under the action of an one-parameter group of unitary transformations.

In order to facilitate comparison, let us gather here the basic principles of the theory of quantum processes:

E1.Observables and events: A physical system is characterised by a history space $\Omega$, that is a suitable subset of a Cartesian product $\times_{t} \Gamma_{t}$, for some manifold $\Gamma$. Events correspond to Borel subsets of $\Omega$ and observables to functions on $\Omega$.

E2. States: The decoherence functional is a bilinear functional on $\Omega$ satisfying properties C1-C6.

E3. Probabilities and phases: If $A$ and $B$ are two histories (subsets of $\Omega$ ) then $\Phi(A, A)$ gives the probability that $A$ is true and $\Phi(A, B)$ gives the relative Pancharatnam phase, measured in the way described in section 2.4 .

E4. Combination of subsystems: If $\Omega_{1}$ and $\Omega_{2}$ are describe two physical systems, then $\Omega_{1} \times \Omega_{2}$ describes the combined system.

E5. Conditioning: One conditions with respect to a subalgebra using the conditional pair and incorporates information about previous experiments through the conditional correlation (see section 3.4.2).

Let us now recall the results of section 5. Principles D1, D2 arise in quantum processes that satisfy the Markov, time-reversibility and time-homogeneity properties. Principle D3 is the one where the two theories disagree and we shall examine it in more detail in a moment. Principle D4 is equivalent to E3, when the decoherence functional is restricted to a moment of time. 
Concerning the combination of subsystems, let us note the following. If $\Gamma=$ $\Gamma_{1} \otimes \Gamma_{2}$ is the phase space at a moment of time in a Markov process the space $V=$ $\mathcal{L}^{2}\left(\Gamma, d z_{1} d z_{2}\right)$ naturally appears and is isomorphic to $\mathcal{L}^{2}\left(\Gamma_{1}, d z_{1}\right) \otimes \mathcal{L}^{2}\left(\Gamma_{2}, d z_{2}\right)=$ $V_{1} \otimes V_{2}$. Now if the two processes are independent, the decoherence functional will be $\Phi_{1} \otimes \Phi_{2}$, which in a Markov process implies that both the initial state $v_{0}$ and the propagator $\chi$ factorises as

$$
\chi_{t}\left(z_{1}, z_{2} \mid z_{1}^{\prime}, z_{2}^{\prime}\right)=\chi_{1 t}\left(z_{1} \mid z_{1}^{\prime}\right) \chi_{2 t}\left(z_{2} \mid z_{2}^{\prime}\right) .
$$

Clearly the projector $E$ that corresponds to the kernel $\psi=\lim _{t \rightarrow 0} \chi_{t}$ also factorises and the resulting physical Hilbert space can be written as $H=H_{1} \otimes$ $H_{2}$. Note that if the two subsystems are not independent, the physical Hilbert space is still a subspace of $V_{1} \otimes V_{2}$.

As we discussed in 3.4.2 the notion of the conditional correlation recovers the standard results for the "reduction of the wave packet " rule D6. And finally a time-reversible, Markov quantum process can be described by an one parameter family of unitary operators. If the process is also time-homogeneous, then this family of operators is an one-parameter group.

\subsection{Do we measure operator's eigenvalues?}

So the only difference between the theory of quantum processes and quantum mechanics is the principle D3: in a quantum process the spectrum of an operator is simply not relevant to the values of the corresponding observable, because at the fundamental level observables are functions on the history space $\Omega$. Clearly there is little difference as far as observables with continuous spectrum are concerned (position, momentum etc). The difference lies, of course, in the case of observables with discrete spectrum.

The case of discrete spectrum is, in fact, what has given quantum phenomena their name, as it is this through the discrete spectrum of operators that the paradigmatic quantum behaviour is manifested: historically it was the black body radiation, the photoelectric effect and the Bohr's atom transitions that put discreteness as a basic feature of the new mechanics. For this reason postulate D3 was highlighted in all early work of quantum theory: it provided a simple solution to the problems that had faced a generation of physicists. Later mathematical development -namely the spectral theorem - offered this postulate the additional justification of mathematical elegance.

It would seem that this is one of the most solid postulates of quantum mechanics, the last one to be taken away from any possible modification of the theory. After all it provides the solution to the physical puzzles that led to quantum mechanics. However, as we are going to argue it is the postulate of quantum theory that is least justified empirically, when taken by itself.

To see this we shall consider the case of atom spectroscopy, which has been historically the main arena justifying the postulate D3. When we study the 
electromagnetic radiation emitted from atoms, we see that the intensity of the electromagnetic field has peaks in particular discrete values of the frequency. Then assuming energy conservation, the photons are viewed as arising from a transition between two "states" of an atom, each of which is characterised by a sharp value of energy. The fact that we measure a number of sharp peaks rather than a smoother distribution of field intensity plotted versus frequency, leads to the conclusion that the possible values of atom's energy are discrete. If we assume that this experiment measures the atom's energy, then this takes discrete values, something that is naturally explained in terms of postulate D3: in any individual measurement only points of the spectrum of the operator are obtained.

We believe that this is a fair summary of the argument that leads to the acceptance of the postulate D3 in the particular context. We shall now see, that the conclusions of the argument is by no means necessary. Let us first make the too obvious remark, that the intensity peaks have finite width and are not sharp. The width is due not only to experimental errors, but comes fundamentally from the time-energy uncertainty relation. Hence, it is only in an idealisation that the atom's energy values are discrete.

However, the most important argument is that the description in terms of atom transitions is semiclassical rather than quantum. What we measure in spectroscopy is the energy/frequency of the electromagnetic field. We typically assume that the emitted photons are incoherent (both in the classical and the quantum sense), so that the emitted electromagnetic field can be considered as an ensemble of photons. Then, we can idealise the experiments by means filters that allow only very narrow frequency (energy) range to pass and measure the intensities. The whole experiment is then fully described by energy measurements of the photons. One can give an equivalent description in terms of the electromagnetic fields. So the actual observables that correspond to the set-up of the experiment is photon energies or fluxes, not atomic energies. And these energies can be described by continuous variables in either quantum theory or in the quantum process description.

The attribution of discrete energy values to the atom comes from a semiclassical picture of the atom/field interaction; it involves a mixture of old quantum theory concepts (orbitals, transitions), with the framework of mature quantum theory. This picture is helpful for calculations, it provides an intuitive picture of the interaction, but it is not fundamentally quantum mechanical. A precise treatment ought to consider the combined system field-atom, interacting perhaps through QED and then consider energy measurements of the electromagnetic field at particular spatial locations. In such a description all information about the process (including the atom's eigenvalues) would be found in the correlation functions of the electromagnetic field: but these are predicted by quantum processes in full agreement with standard quantum theory.

What we imply by this argument, is that historically the discrete values of observables refer to the spectrum of the Hamiltonian, rather than any arbi- 
trary observable. The information about its eigenvalues is fully contained in the correlation functions: once these are provided, we can read off any discretised behaviour. In other words, the discrete behaviour in quantum theory is not fundamental, but arises due to particular forms of the dynamics. This is true even for spin systems: the "discrete" spin values are always measured in conjunction with its coupling to some magnetic field.

Discrete behaviour arising from dynamics is not something strange or new. The stochastic mechanics developed by Nelson [43] is a framework trying to describe quantum theory in term of stochastic processes on configuration space: as in our case, stochastic mechanics considers fundamentally continuous observables and generates discrete structures dynamically. Stochastic mechanics ultimately fails as an alternative theory to quantum mechanics, since it cannot account for Bell's theorem, however it has provided many examples by which stochastic process simulate discrete behaviour, actually reproducing quantum mechanical phenomenology. The reader is referred to $[43,44]$ for elaboration of arguments similar to the ones we presented here.

We conclude our argument by noticing, that of all axioms of Kopenhagen quantum theory, it is only D3 that makes implicit reference to properties of individual quantum systems (namely in any individual system we measure certain particular values for an observable). If we take the stance that Kopenhagen is a statistical theory of ensembles, axiom D3 might be viewed as the odd one among all quantum mechanical postulates.

\subsection{Preparation and conditioning}

Another point we want to address is the notion of preparation. The controlled preparation of quantum state is the first important part of any measurement scheme. There are various procedures that are followed in actual experiments: these procedures have to be repeatable and aim to provide an accurate description of the system in terms of a well specified Hilbert space vector or density matrix.

In the theory of quantum processes one represents the effects of preparation by the notion of conditioning. We need first to assume a basic decoherence functional for the system: this is not the effective decoherence functional we employ for the measurements, it is just a decoherence functional representing the systems under study before even preparation. Its actual form will probably play little role in the set-up of the measurement process. Thus it can be a decoherence functional of the standard form, with initial density matrix corresponding to total ignorance; or it can be of a simple factorised form

$$
\Phi(A, B)=\sigma(A) \bar{\sigma}(B) .
$$

Whatever the initial form might be, as soon as the experimentalist starts interfering with the system, he will not employ the initial decoherence functional in his calculations. Rather he has to transform the initial decoherence 
functional through the incorporation of all information corresponding to the preparation procedure. If, for instance, the experimentalist passes a beam of particles through a filter so that only ones with a given property will be further studied, the decoherence functional has to transform according to the standard rules.

The preparation of the system is then described by successive conditionings of the decoherence functional, plus evolution that needs be neither unitary (systems are often coupled to external reservoirs so that they relax in their ground state), or even Markovian (the experimentalist can choose to perform or not a given operation according to the outcome of a past preparation procedure).

In principle, one can describe the whole sequence of preparation procedures, either as conditioning of the decoherence functional, or introduction of "unusual" dynamics at different times $t$. However, physical systems seem to be described (when isolated) by Markovian dynamics, so at the moment $t=0$, where the last step of preparation has been concluded, the whole information of the preparation can be encoded in its restriction at $t=0$ (in other words a density matrix) and its evolution operator: the process is then Markovian for all times $t \geq 0$; and naturally it is not Markovian if the whole of the time axis is taken as describing it.

We should keep in mind, that if the physical systems were not described by a Markov process, the description in terms of an initial state would be meaningless, since any step in the preparation procedure might affect (unexpectedly) later evolution of the system.

The other point to note, is that typical preparation procedures aim to force the quantum system to forget its past or to make the past irrelevant for the experimental procedure: the coupling to a reservoir, for instance, brings all systems to their initial state. So it seems fair to assume that the initial form of the decoherence functional before conditioning, will be largely irrelevant to the determination of the density matrix that arises out of the preparation. In this context, the question whether there is some decoherence functional describing the system outside operational situations can have no answer; at least not until we have an acceptable quantum theory for individual systems.

\subsection{Further remarks}

As far as the interpretation of quantum theory is concerned, the theory of quantum processes has a feature, which we think is a distinct advantage over standard quantum theory. Its "logic" is classical. Even though we have cast the theory in a Kopenhagen form, there is no problem in talking about properties of individual systems outside a measurement context (there is no problem of definability), as long as we remember that all our predictions refer to ensembles. This theory completely sidesteps the obstacles of theorems such as the one of Kochen and Specker for the existence of uncontextual realist theories. A full discussion about these issues is to be found in [12]. 
In the same reference it is also discussed, whether the difference between quantum processes and standard quantum theory in terms of the notion of event can be experimentally determined. The two theories differ in their statistical correlation functions (2. 6), while they have the same quantum mechanical correlation functions. The answer tends to be negative, even though the possibility of devising an experiment that distinguishes them cannot be ruled out. The reason is -in a sentence- that in realistic experiments the actual time evolution (due also to the coupling of the system with the measuring device) tends to blur the distinction, because quantum mechanical time evolution does not preserve the characteristic functions on the phase space. The reader is again referred to [12] for a fuller analysis.

\section{Conclusions}

In this paper we described a theoretical framework for quantum theory, which is distinct from standard quantum theory. It is a development of the following basic ideas

i. Quantum theory provides information not only about probabilities but also of complex phases relative to different histories: these phases are measurable in the same way as the Pancharatnam phase. The information about both phases and probabilities is encoded in the decoherence functional, which also contains all information about the system's correlation functions.

ii. Putting complex phases as primitive ingrdients of theory leads to a description of a system in terms of non-Kolmogorov probability. All constraints coming from non-go theorems do not hold here, hence we can write a theory with commutative observables (i.e. "hidden variables") that can reproduce the results of standard quantum theory.

iii. The classical phase space of the system contains enough information, to fully incorporate all quantum mechanical behaviour. Using the formal analogy with stochastic processes, we can set up a theory of quantum processes on phase space: the basic object will be now the decoherence functional rather than the probability measure.

We then saw, how this construction can be developed and recover standard quantum theory, by demanding the satisfaction of the Markov property and that the kinematic process is not trivial.

What do we think are the basic achievements of this paper?

We showed, that there is the possibility of a different axiomatic framework for quantum theory, that does not necessitate the introduction of non-commutative objects as fundamental. The distinctive quantum mechanical behaviour is contained in an object that plays the role of the density matrix for histories: the decoherence functional. In our construction, there is no need to consider that 
quantum theory necessitates a radical change in the concept of events (as quantum logic is often taken to imply). Our logic remains Boolean. We can say that we have a theory of hidden variables; they are non-deterministic, of course, but they are fully compatible with realism.

We could then come forward and claim that the theory of quantum processes provides an alternative description of quantum phenomena, from which the Hilbert space description arises as a convenient special case. Our arguments were completely general and we did not specialise on any particular system: it suffices that one can write an effective phase space description for the quantum system. Our result are valid also for bosonic fields, but they are not readily employed for spinor fields. The reason is that, as yet we lack a phase space description of spinor fields (through coherent stace) that corresponds to a theory with local Hamiltonian (the existing fermionic coherent states [45] have non-local dynamics). We are trying to address the issue by constructing coherent states for the fermion fields, starting from coherent states of the corresponding relativistic particles. With the same motivation we have provided a relevant discussion for the spin-statistics theorem [46]. If we succeed in this endeavour, we shall be in a position to claim that a description of quantum processes can supplement standard quantum theory in all physical systems accessible to experiment.

One could then raise the issue of naturality: how natural is the construction of quantum processes as compared to standard quantum theory? This is a difficult question to answer: by what criteria is a notion of a functional containing phase and probability information more (or less) natural than the notion that observables are actually non-commutative objects? What is natural is often an issue of universality, but sometimes it is simply a matter of habit.

Our eventual aim is, however, different from either the interpretational issue or the rather interesting mathematical constructions a theory of quantum processes can lead to. After all, we do not provide different domain of applicability from standard quantum theory. Now, if we accept that the notion of events in quantum theory is not different from the one of classical theory, there is a natural question to ask. We have a successful statistical theory for physical systems that gives non-additive probabilities, which are related to some relative $U(1)$ phases of unknown origin; can we explain this statistical behaviour in terms of properties of the individual system? To lead our investigations towards the construction of such a theory, is the final aim of this paper.

\section{References}

[1] R. Griffiths, Consistent Histories and the Interpretation of Quantum Mechanics, J. Stat. Phys. 36, 219, 1984.

[2] R. Omnès, Logical Reformulation of Quantum Mechanics: I Foundations, J. Stat. Phys. 53, 893, 1988; The Interpretation of Quantum Mechanics, 
(Princeton University Press, Princeton, 1994); Consistent Interpretations of Quantum Mechanics, Rev. Mod. Phys. 64, 339, 1992.

[3] M. Gell-Mann and J. B. Hartle, Quantum mechanics in the Light of Quantum Cosmology, in Complexity, Entropy and the Physics of Information, edited by W. Zurek, (Addison Wesley, Reading, 1990); Classical Equations for Quantum Systems, Phys. Rev. D 47, 3345, 1993.

[4] J. B. Hartle, Spacetime Quantum Mechanics and the Quantum Mechanics of Spacetime, in Proceedings on the 1992 Les Houches School, Gravitation and Quantisation, 1993.

[5] C.J. Isham, Quantum Logic and the Histories Approach to Quantum Theory, J. Math. Phys. 35, 2157, 1994.

[6] M. V. Berry. Quantal Phase Factors Accompanying Adiabatic Changes, Proc. Roy. Soc. Lond. A392:45, 1984.

[7] B. Simon. Holonomy, the Quantum Adiabatic Theorem and Berry's Phase. Phys. Rev. Lett. 51: 2167, 1983.

[8] A. Shapere and F. Wilczek (editors), Geometric Phases in Physics, (World Scientific, Singapore, 1989).

[9] Y. Aharonov and J. Anandan. Phase Change During a Cyclic Quantum Evolution, Phys. Rev. Lett. 58: 1593, 1987.

[10] J. Samuel and R. Bhandari, General Setting for Berry Phase, Phys. Rev. Lett. 60, 2339, 1988.

[11] C. Anastopoulos and N. Savvidou, Quantum Mechanical Histories and the Berry Phase, Int. J. Th. Phys. 41, 529, 2002.

[12] C. Anastopoulos, Quantum Theory without Hilbert Spaces, Found. Phys. 31, 1545, 2001.

[13] , S. Pancharatnam, , Proc. Ind. Acad. Sci. A 44, 246, 1956.

[14] J. S. Bell, "On the Einstein-Podolsky-Rosen Paradox", Physics 1, 195, 1964.

[15] S. Kochen and R. P. Specker, "The Problem of Hidden Variables in Quantum Mechanics", J. Math. Mech. 17, 59, 1967.

[16] R. F. Streater, Classical and Quantum Probability, J. Math. Phys. 41, 3556, 2000 .

[17] K. Savvidou, The Action Operator in Continuous Time Histories, J. Math. Phys. 40, 5657, 1999. 
[18] N. Savvidou, Poincaré invariance for Continuous-Time Histories, J. Math. Phys. 43,3053 (2002).

[19] N. Savvidou, General Relativity Histories Theory: Spacetime Diffeomorphisms and the Dirac Algebra of Constraints, Class. Quant. Grav. 18, 3611, 2001.

[20] C. DeWitt- Morette, K. D. Elworthy (ed.), New Stochastic methods in Physics, Phys. Rep. 77, 121, 1981.

[21] W. Horsthemke and R. Lefever, Noise induced Processes: Theory and Applications in Physics, Chemistry and Biology, (Springer, Berlin, 1984).

[22] R. F. Streater, Statistical Dynamics , (Imperial College Press, London, 1996).

[23] N. Savvidou, Continuous Time in Consistent Histories, gr-qc/9912076.

[24] C.J.Isham and N. Linden. Continuous Histories and the History Group in Generalised Quantum Theory, J. Math. Phys. 36: 5392, 1995.

[25] C. Isham, N. Linden, K. Savvidou and S. Schreckenberg, "Continuous Time and Consistent Histories", J. Math. Phys. 37, 2261, 1998.

[26] C. Anastopoulos, Continuous-time Histories: Observables, Probabilities, Phase Space Structure and the Classical Limit, J.Math.Phys. 42, 3225, 2001.

[27] C. Anastopoulos, Quantum Correlation Functions and the Classical Limit, Phys. Rev. D63, 125024, 2001.

[28] A. Wagh, V. C. Rakhecha, P. Fischer and A. Ioffe, Neutron Interferometric Observation of Noncyclic Phase, Phys. Rev. Lett. 1992, 1998.

[29] J. S. Schwinger, Brownian Motion of a Quantum Oscillator, J. Math. Phys. 2, 407 (1961).

[30] L. V. Keldysh, Diagram Technique for Nonequilibrium Processes, Zh. Eksp. Teor. Fiz. 47, 1515, 1964.

[31] C.J. Isham and N. Linden, Quantum Temporal Logic and Decoherence Functionals in the Histories Approach to Generalised Quantum Theory, J. Math. Phys. 35, 5452, 1994.

[32] C. J. Isham, Topological and Global Aspects of Quantum Theory, in Proceedings of the 1983 Les Houches School, Relativity, Groups and Topology II.

[33] M.D. Srinivas. Quantum Mechanics as a Generalized Stochastic Process on Phase Space, Phys. Rev. D15: 2837, 1977. 
[34] H. Grabert, P. Hänggi and P. Talkner, Is Quantum Mechanics Equivalent to a Classical Stochastic Process?, Phys. Rev. A 19, 2440, 1979.

[35] W. Heisenberg: Collected Works, Series A, Part I, edited by W. Blum, H.-P. Durr, and H. Rechenberg (Springer Verlag, Berlin, 1984).

[36] J. R. Klauder and B. Skagerstam (editors), Coherent States: Applications in Physics and Mathematical Physics, (world Scientific, SIngapore, 1985).

[37] A. Perelomov, Generalized Coherent States and their Applications, (Springer, Berlin, 1986).

[38] N. Mukunda and R. Simon, Quantum Kinematic Approach to the Geometric Phase: 1. General Formalism, Ann. Phys. 228, 205, 1993.

[39] E. B. Davies, Quantum Theory of Open Systems, (Academic Press, London, 1976).

[40] R. Omnés, Logical Reformulation of Quantum Mechanics: 4. Projectors in Semiclassical Physics, J. Stat. Phys. 57, 357, 1989.

[41] C. Anastopoulos, Quantum Fields in Non-Static background: a Histories Perspective, J. Math. Phys. 41, 617, 2000.

[42] J. R. Klauder, Quantization is Geometry, After All, Ann. Phys. 188, 120, 1988; Geometric Quantization from a Coherent State Viewpoint, quant$\mathrm{ph} / 9510008$.

[43] E. Nelson, Quantum Fluctuations, (Princeton University Press, Princeton, 1985).

[44] G. C. Ghirardi, C. Omero, A. Rimini, T. Weber, The Stochastic Interpretation of Quantum Mechanics: a Critical Review, Riv. Nuovo Cim. 1N3, 1, 1978.

[45] J. Klauder. The Action Option and a Feynman Quantization of Spinor Fields in terms of Ordinary C- Numbers, Ann. Phys 11, 123, 1959.

[46] C. Anastopoulos, Spin-Statistics Theorem and Geometric Quantisation, quant-ph/0110169. 